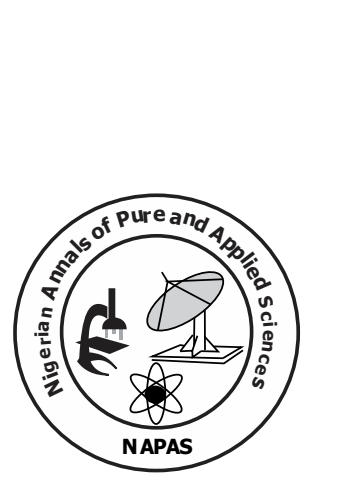
s:

\section{A Mathematical Model of Stratified Geophysical Fluid Flows over Variable Bottom Topography}

\section{lornumbe, S.I. ${ }^{1}$, Tivde T.2; and Chia, R.A. ${ }^{1}$}

1 Department of Mathematics and Computer Science Benue State University, Makurdi

2Federal University of Agriculture, Makurdi, Benue State

Corresponding author:iorsteve@yahoo.com.

\begin{abstract}
In this paper, a mathematical model of stratified geophysical fluid flow over variable bottom topography was derived for shallow water. The equations are derived from the principles of conservation of mass and conservation of momentum. The force acting on the fluid is gravity, represented by the gravitational constant $g$. A system of six nonlinear partial differential equations was obtained as the model equations. The solutions of these models were obtained using perturbation method. The presence of the coriolis force in the shallow water equations were shown as the causes of the deflection of fluid parcels in the direction of wave motion and causes gravity waves to disperse. As water depth decreases due to varied bottom topography, the wave amplitude were shown to increase while the wavelength and wave speed decreases resulting in overturning of the wave. The results are presented graphically.
\end{abstract}

Keywords: Bottom topography, coriolis force, geophysical fluid, series solution, shallow water equations, stratification. 


\section{Introduction}

Stratified fluids are very present in nature, present in almost any heterogeneous fluid body. Examples include thermal stratification of reservoirs and oceans, salinity stratification in estuaries, rivers, groundwater reservoirs, and oceans, heterogeneous mixtures in industrial, food, and manufacturing processing, density stratification of the atmosphere and many others. In the presence of gravity, these density differences have a dramatic impact on the dynamics and mixing of heterogeneous fluids. For instance, thermal stratification in reservoirs can reduce the vertical mixing of oxygen to the point that bottom water becomes anoxic through the action of biological processes. Preventing, predicting, and solving such reservoir problem requires an understanding of the dynamics of stratified fluids. Therefore a stratified fluid is one in which the fluid density $\rho$ is a function of space $(x, y, z)$ and time $t$. The term stratification refers to the variation in the density field. Density differences arise through many sources. These can include differences in temperature, dissolved phases (solids, liquids, and gases), suspended solids, and pressure differences. The development of layers within the profile of a water body is a common phenomenon of stratification, (Pedlosky, 1987).

There are vast numbers of publications covering the different fields of classical geophysical fluid dynamics. General reviews and background information may be found in several textbooks; (Gill 1982; Mellor 1996; Pedlosky 1987 and Perks 1988). In the present context, with emphasis on more local scale geophysical flows, we may consider the most relevant literature in waterly flows as follows: for local waterly flows, analysis and laboratory experiments may be found in (Boyer and Davies 2000, Riley and Lelong 2000), while modeling and applications ranging from local tidal flow to circulations in various restricted waters are given in (Moe et al., 2002).

Dellar and Salmon (2005) derived an extended set of shallow water equations that describe a thin inviscid fluid layer above fixed topography in a frame rotating about an arbitrary axis and also derived models that treat the ocean or atmosphere as a stack of layers with variable thickness. Within each layer, the density is either assumed to be uniform or may vary horizontally due to temperature gradients.

According to Karelsky et al. (2000), the generalization of classical shallow water theory to the case of flows over an irregular bottom. They showed that the simple self-similar solutions that are characteristic for the classical problem exist only if the underlying surface has a uniform slope.

A mathematical simulation to determine the water velocity in the Lake Mariut, taking into consideration its concentration and the distribution of the temperature along it, by applying the fractional steps method for the numerical solution of the shallow water equations was developed in (Abd-el-Malek et al., 2007). The 2D shallow water equations was used to describe water flows with a free surface under the influence of gravity (with gravitational acceleration ) and the Coriolis force due to the earth's rotation with angular velocity, (Vallis, 2006). The shortcoming of the model is that it does not take into account the density stratification which is present in the geophysical flow. Iornumbe et al.,(2020) considered the model of the bottom topography of a geophysical fluid flow in the presence of Coriolis force, without stratification and a system of three nonlinear partial differential equations in two dimensions was modelled and solved using the perturbation method. Since stratified fluids are very present in nature, present in almost any heterogeneous fluid body, therefore, we considered it in this paper.

In this paper, a mathematical model for the stratified geophysical fluid flow over variable bottom topography is derived taken into account the density stratification which is present in the geophysical flow and is an extension of the work of Iornumbe et al. (2020). 


\section{Model Formulation}

\section{Model Assumptions}

In formulating the models, we make the following assumptions:

1. The flow is predominantly horizontal, and the vertical acceleration is small compared to the gravity acceleration.

2. The Cartesian coordinates $x, y$ and $z$ will be used, with $z$ measured vertically upward

3. The velocity components in the directions of increasing $x, y$ and $z$ will be denoted by $u, v$ and $w$

4. Take the $(x, y)$ horizontal plane as being parallel to the surface of the still water, and the depth of the water at a given point as $h=h(x ; y ; t)>0$.

5. We denote velocity in the $x$-direction as $u=u(x ; y ; t)$ and the velocity in the $\mathrm{y}$ direction as $v=v(x ; y ; t)$. While the plane $(z=0)$ can be chosen arbitrarily, it is usually positioned at mean water level.

6. Measuring down from this plane, the bottom of the harbour is at depth $z=-\zeta(x, y)$. The equation $z=-\zeta(x, y)$ is the equation for the bottom surface, also known as the variable bottom topography or bathymetry, the depth of which is usually assumed to vary with $x$ and $y$.

7. The stratified fluid is one in which the fluid density $\rho$ is a function of space $(x, y, z)$ and time $t$

\section{Model Equations}

According to Iornumbe et al. (2020); the momentum and Continuity equation for the two-dimensional shallow water flow model taking into account the effects of topography and the Earth's rotation was described as below:

The momentum equations in the two directions are;

$$
\begin{aligned}
& \frac{\partial(h u)}{\partial t}+\frac{\partial\left(u^{2} h+g h^{2} / 2\right)}{\partial x}+\frac{\partial(h u v)}{\partial y}=-g h \frac{\partial \xi}{\partial x}+f v \\
& \frac{\partial(h v)}{\partial t}+\frac{\partial(h u v)}{\partial x}+\frac{\partial\left(v^{2} h+{ }^{g h^{2}} / 2\right)}{\partial y}=-g h \frac{\partial \xi}{\partial y}-f u
\end{aligned}
$$

And the Continuity Equation as;

$$
\frac{\partial h}{\partial t}+\frac{\partial(h u)}{\partial x}+\frac{\partial(h v)}{\partial y}=0
$$

We now consider the dynamics of multiple layers of fluid stacked on top of each other. This is a crude representation of continuous stratification, but it turns out to be a powerful model of many geophysical interesting phenomena. The pressure is continuous across the interface, but the density jumps discontinuously and this allows the horizontal velocity to have a corresponding discontinuity. In each layer pressure is given by the hydrostatic approximation, and so anywhere in the interior. We can find the pressure by integrating the hydrostatic approximation down from the top. Thus, hydrostatic approximation is,

$\frac{d p}{d z}=-g \rho$ 

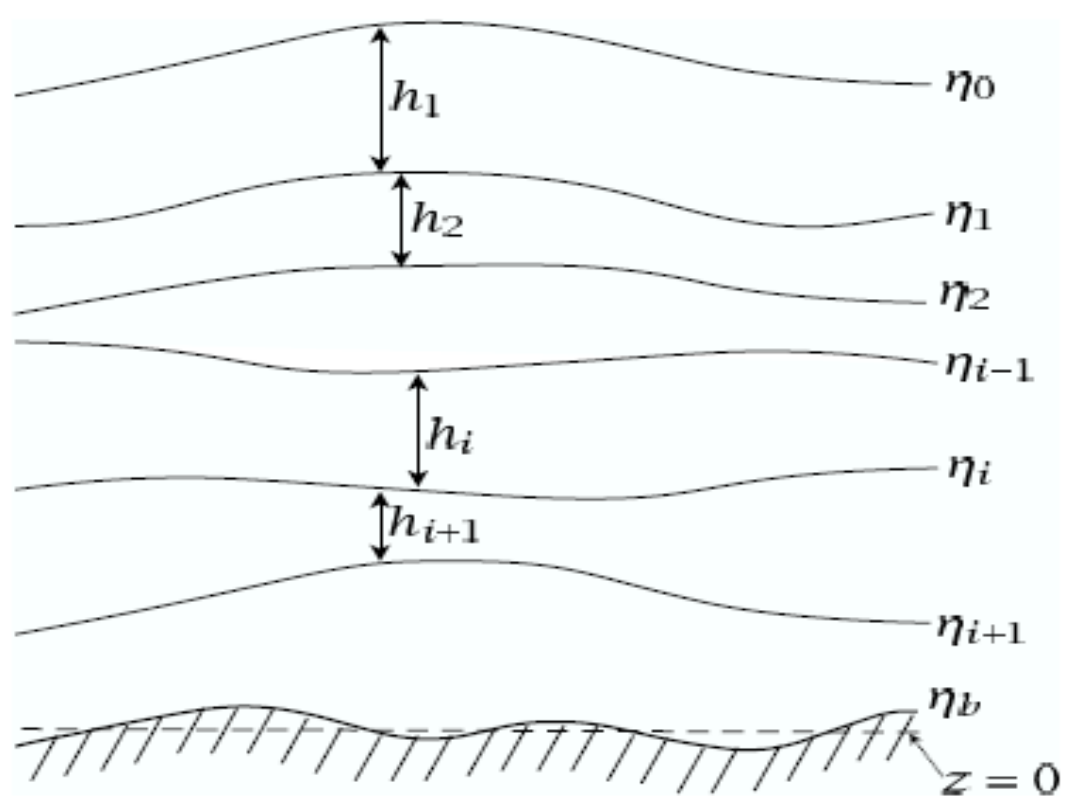

Figure 1: The multilayer stratified shallow water system. The layers are numbered from the top down. The coordinates of the interfaces are denoted by $\eta$, and the layer thicknesses $h_{i}$.

Integrating equation (4) from $\eta_{0}$ to $z$

$\int d p=\int_{\eta_{0}}^{z}-g \rho d z$

$p_{1}=-\rho_{1} g\left(z-\eta_{0}\right)$

$p_{1}=\rho_{1} g\left(\eta_{0}-z\right)$

For the second layer, integrating from $\eta_{0}$ to $\eta_{1}$ and $\eta_{1}$ to $z$ we have

$p_{2}=-\rho g \int_{\eta_{0}}^{\eta_{1}} d z-\rho g \int_{z}^{\eta_{1}} d z$

$p_{2}=\rho_{1} g\left(\eta_{0}-\eta_{1}\right)+\rho_{2} g\left(\eta_{1}-z\right)$

$p_{2}=\rho_{1} g \eta_{0}+\rho_{1} g_{1}^{\prime} \eta_{1}-\rho_{2} g z$

where

$g_{1}^{\prime}=g \frac{\left(\rho_{2}-\rho_{1}\right)}{\rho_{1}}$. These results obtain

similarly for other levels. The term involving $z$ is irrelevant for the dynamics because only the horizontal motion is considered. Omitting this term for the two layered model gives the dynamical pressure as;

$\left.\begin{array}{l}p_{1}=\rho_{1} g \eta_{0} \\ p_{2}=\rho_{1} g \eta_{0}+\rho_{1} g_{1}^{\prime} \eta_{1}\end{array}\right\}$

$5 \quad \eta_{i}$ can be summed from the top down; hence

$\eta_{0}=h_{1}+h_{2}+\eta_{b}$ and $\eta_{1}=h_{2}+\eta_{b}$

Therefore the pressure in the two layers' system can be express as;

$\left.\begin{array}{l}p_{1}=\rho_{1} g \eta_{0}=\rho_{1} g\left(h_{1}+h_{2}+\eta_{b}\right) \\ p_{2}=\rho_{1} g \eta_{0}+\rho_{1} g_{1}^{\prime} \eta_{1}=\rho_{1} g\left(h_{1}+h_{2}+\eta_{b}\right)+\rho_{1} g_{1}^{\prime}\left(h_{2}+\eta_{b}\right)\end{array}\right\}^{8}$

Finally, the mass conservation equation for each layer has the same form as,

$\frac{D h_{n}}{D t}+h_{n} \nabla \cdot u_{n}$ 


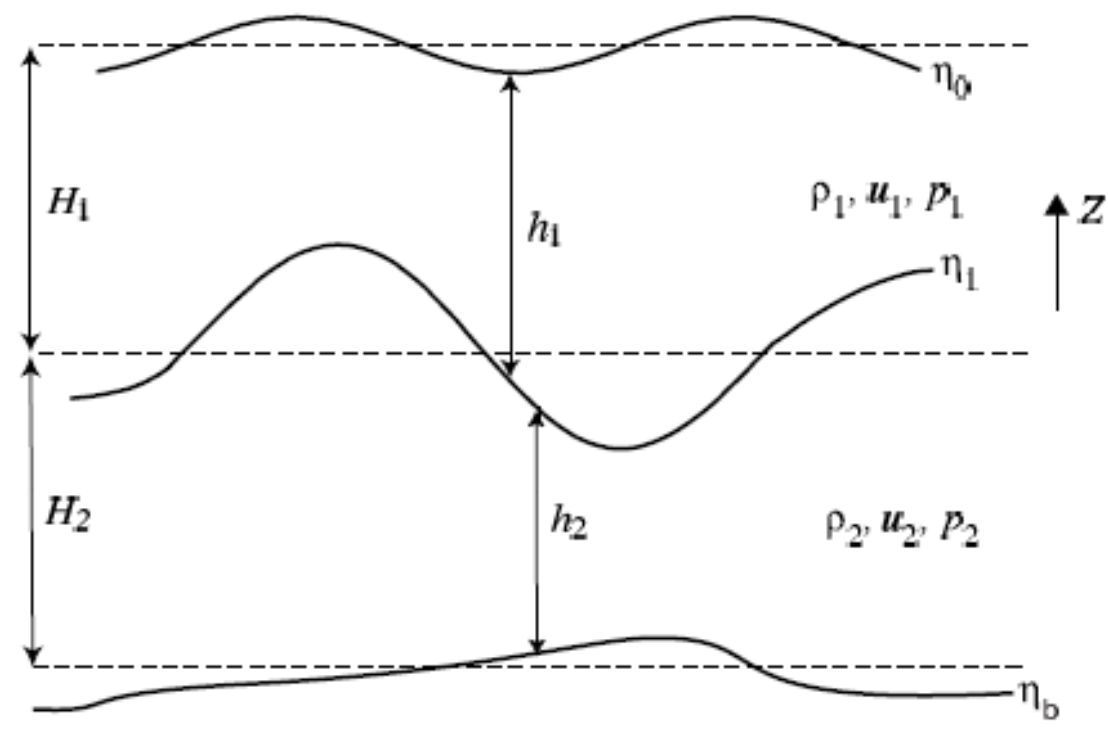

Figure 2: The two layered-stratified shallow water system with varied bottom topography. A fluid of density $\rho_{1}$ lies over a denser fluid of density $\rho_{2}$.

For three layer model, pressure can be given as;

$$
\left.\begin{array}{l}
p_{1}=\rho_{1} g \eta_{0}=\rho_{1} g\left(h_{1}+h_{2}+h_{3}+\eta_{b}\right) \\
p_{2}=\rho_{1} g \eta_{0}+\rho_{1} g_{1}^{\prime} \eta_{1}=\rho_{1} g\left(h_{1}+h_{2}+h_{3}+\eta_{b}\right)+\rho_{1} g_{1}^{\prime}\left(h_{2}+h_{3}+\eta_{b}\right) \\
p_{3}=\rho_{1} g \eta_{0}+\rho_{1} g_{1}^{\prime} \eta_{1}+\rho_{1} g_{2}^{\prime} \eta_{2}=\rho_{1} g\left(h_{1}+h_{2}+h_{3}+\eta_{b}\right)+ \\
\rho_{1} g_{1}^{\prime}\left(h_{2}+h_{3}+\eta_{b}\right)+\rho_{1} g_{2}^{\prime}\left(h_{3}+\eta_{b}\right)
\end{array}\right\}
$$

with

$\eta_{i}=\eta_{b}+\sum_{i=n+1}^{i=N} h_{i}$

where $N$ is the $\mathrm{n}^{\text {th }}$ layer model, and " $n$ " is the position of the layer in the multiple layers system. Therefore for the $\mathrm{n}^{\text {th }}$ layer model, the dynamical pressure is given as;

$p_{n}=\rho_{i} \sum_{i=0}^{n-1} g_{i}^{\prime} \eta_{i}$

where

$g_{i}^{\prime}=g \frac{\left(\rho_{i+1}-\rho_{i}\right)}{\rho_{i}} \quad$ and taking $\rho_{0}=0$

Therefore the two-layer models of geophysical fluid flows over variable topography are given by combining $(1),(2),(3)$ and $(8)$ 


$$
\left.\begin{array}{l}
\frac{\partial\left(h_{1} u_{1}\right)}{\partial t}+\frac{\partial\left(h_{1} u_{1}^{2}+g h_{1}^{2} / 2\right)}{\partial x}+\frac{\partial\left(h_{1} u_{1} v_{1}\right)}{\partial y}=-g h_{1} \frac{\partial h_{2}}{\partial x}-g h_{1} \frac{\partial(\xi)}{\partial x}+f v_{1} \\
\frac{\partial\left(h_{1} v_{1}\right)}{\partial t}+\frac{\partial\left(h_{1} u_{1} v_{1}\right)}{\partial x}+\frac{\partial\left(h_{1} v_{1}^{2}+g h_{1}^{2} / 2\right)}{\partial y}=-g h_{1} \frac{\partial h_{2}}{\partial y}-g h_{1} \frac{\partial(\xi)}{\partial y}-f u_{1} \\
\frac{\partial h_{1}}{\partial t}+\frac{\partial\left(h_{1} u_{1}\right)}{\partial x}+\frac{\partial\left(h_{1} v_{1}\right)}{\partial y}=0 \\
\frac{\partial\left(h_{2} u_{2}\right)}{\partial t}+\frac{\partial\left(h_{2} u_{2}^{2}+g h_{2}^{2} / 2+g h_{2} h_{1}\right)}{\partial x}+\frac{\partial\left(h_{2} u_{2} v_{2}\right)}{\partial y}=-g h_{1} \frac{\partial h_{2}}{\partial x}-g h_{2} \frac{\partial(\xi)}{\partial x}+f v_{2} \\
\frac{\partial\left(h_{2} v_{2}\right)}{\partial t}+\frac{\partial\left(h_{2} u_{2} v_{2}\right)}{\partial x}+\frac{\partial\left(h_{2} v_{2}^{2}+g h_{2}^{2} / 2+g h_{2} h_{1}\right)}{\partial y}=-g h_{1} \frac{\partial h_{2}}{\partial y}-g h_{2} \frac{\partial(\xi)}{\partial y}-f u_{2} \\
\frac{\partial h_{2}}{\partial t}+\frac{\partial\left(h_{2} u_{2}\right)}{\partial x}+\frac{\partial\left(h_{2} v_{2}\right)}{\partial y}=0
\end{array}\right\}
$$

Hence the model is complete for the six variables $h_{1}, u_{1}, v_{1}, h_{2}, u_{2}$ and $v_{2}$ such that the $x$-axis is taken eastward and the $y$-axis is taken northward, with $u$ and $v$ the corresponding velocity components, respectively, $g$ stands for the gravitational constant, $f=2 \Omega \sin \varphi$ is the Coriolis parameter, $\Omega$ is the angular velocity of the earth rotation, $\varphi$ is the geographical latitude of the earth origin coordinate and $(f v,-f u)$ represents the Coriolis acceleration which is produced by the effect of rotation.

\section{Method of Solution}

We will consider the model equations

$$
\begin{aligned}
& \frac{\partial\left(h_{1} u_{1}\right)}{\partial t}+\frac{\partial\left(h_{1} u_{1}^{2}+g h_{1}^{2} / 2\right)}{\partial x}+\frac{\partial\left(h_{1} u_{1} v_{1}\right)}{\partial y}=-g h_{1} \frac{\partial h_{2}}{\partial x}-g h_{1} \frac{\partial(\xi)}{\partial x}+f v_{1} \\
& \frac{\partial\left(h_{1} v_{1}\right)}{\partial t}+\frac{\partial\left(h_{1} u_{1} v_{1}\right)}{\partial x}+\frac{\partial\left(h_{1} v_{1}^{2}+g h_{1}^{2} / 2\right)}{\partial y}=-g h_{1} \frac{\partial h_{2}}{\partial y}-g h_{1} \frac{\partial(\xi)}{\partial y}-f u_{1} \\
& \frac{\partial h_{1}}{\partial t}+\frac{\partial\left(h_{1} u_{1}\right)}{\partial x}+\frac{\partial\left(h_{1} v_{1}\right)}{\partial y}=0 \\
& \frac{\partial\left(h_{2} u_{2}\right)}{\partial t}+\frac{\partial\left(h_{2} u_{2}^{2}+g h_{2}^{2} / 2+g h_{2} h_{1}\right)}{\partial x}+\frac{\partial\left(h_{2} u_{2} v_{2}\right)}{\partial y}=-g h_{1} \frac{\partial h_{2}}{\partial x}-g h_{2} \frac{\partial(\xi)}{\partial x}+f v_{2} \quad(d) \\
& \frac{\partial\left(h_{2} v_{2}\right)}{\partial t}+\frac{\partial\left(h_{2} u_{2} v_{2}\right)}{\partial x}+\frac{\partial\left(h_{2} v_{2}^{2}+g h_{2}^{2} / 2+g h_{2} h_{1}\right)}{\partial y}=-g h_{1} \frac{\partial h_{2}}{\partial y}-g h_{2} \frac{\partial(\xi)}{\partial y}-f u_{2} \quad(e) \\
& \frac{\partial h_{2}}{\partial t}+\frac{\partial\left(h_{2} u_{2}\right)}{\partial x}+\frac{\partial\left(h_{2} v_{2}\right)}{\partial y}=0
\end{aligned}
$$

(a)

with the initial and boundary conditions 


$$
\left.\begin{array}{lll}
u_{1,2}(x, y, 0)=u_{0}, & u_{x, 1,2}(0, y, t)=0, & u_{x, 1,2}(l, y, t)=0 \\
& u_{y, 1,2}(x, 0, t)=0, & u_{y, 1,2}(x, n, t)=0 \\
v(x, y, 0)=v_{0}, & v_{y}, 1,2(x, 0, t)=0, & v_{y 1,2}(x, n, t)=0 \\
v_{x, 1,2}(0, y, t)=0, & v_{x, 1,2}(l, y, t)=0 \\
h_{1}(x, y, 0)=m e^{-s\left(x^{2}+y^{2}\right)}-\alpha \sin (\beta x), & h_{2}(x, y, 0)=1-\alpha \sin (\beta x), & \\
h_{x, 1,2}(0, y, t)=0, h_{x, 1,2}(l, y, t)=0 & h_{y, 1,2}(x, 0, t)=0, & h_{y, 1,2}(x, n, t)=0
\end{array}\right\}
$$

Equation $(c)$ in $(13)$ can be written as

$\frac{d h_{1}}{d t}=-h_{1}\left(\frac{\partial u_{1}}{\partial x}+\frac{\partial v_{1}}{\partial y}\right)$

Equation $(f)$ in $(13)$ can be written as

$\frac{d h_{2}}{d t}=-h_{2}\left(\frac{\partial u_{2}}{\partial x}+\frac{\partial v_{2}}{\partial y}\right)$

Equation $(a)$ in $(13)$ can be written as:

$\left.\begin{array}{l}u_{1}\left(\frac{\partial h_{1}}{\partial t}+u_{1} \frac{\partial h_{1}}{\partial x}+v_{1} \frac{\partial h_{1}}{\partial y}\right)+h_{1}\left(\frac{\partial u_{1}}{\partial t}+u_{1} \frac{\partial u_{1}}{\partial x}+v_{1} \frac{\partial u_{1}}{\partial y}\right)+h_{1} u_{1}\left(\frac{\partial u_{1}}{\partial x}+\frac{\partial v_{1}}{\partial y}\right)+ \\ g h_{1}\left(\frac{\partial h_{1}}{\partial x}+\frac{\partial h_{2}}{\partial x}\right)=-g h_{1} \frac{\partial \xi}{\partial x}+f v_{1}\end{array}\right\}$

Using (15) in (17), we obtain

$h_{1} \frac{d u_{1}}{d t}+h_{1} g\left(\frac{\partial h_{1}}{\partial x}+\frac{\partial h_{2}}{\partial x}\right)=-h_{1} g \frac{\partial \xi}{\partial x}+f v_{1}$

Equation $(b)$ in $(9)$ can be written as:

$\left.\begin{array}{l}v_{1}\left(\frac{\partial h_{1}}{\partial t}+u_{1} \frac{\partial h_{1}}{\partial x}+v_{1} \frac{\partial h_{1}}{\partial y}\right)+h_{1}\left(\frac{\partial v_{1}}{\partial t}+u_{1} \frac{\partial v_{1}}{\partial x}+v_{1} \frac{\partial v_{1}}{\partial y}\right)+h_{1} v_{1}\left(\frac{\partial u_{1}}{\partial x}+\frac{\partial v_{1}}{\partial y}\right)+ \\ g h_{1}\left(\frac{\partial h_{1}}{\partial y}+\frac{\partial h_{2}}{\partial y}\right)=-g h_{1} \frac{\partial \xi}{\partial y}-f u_{1}\end{array}\right\}$

Using (15) in (19), we obtain

$$
h_{1} \frac{d v_{1}}{d t}+h_{1} g\left(\frac{\partial h_{1}}{\partial y}+\frac{\partial h_{2}}{\partial y}\right)=-h_{1} g \frac{\partial \xi}{\partial y}-f u_{1}
$$

Equation $(d)$ in $(13)$ can be written as: 


$$
\begin{aligned}
& u_{2}\left(\frac{\partial h_{2}}{\partial t}+u_{2} \frac{\partial h_{2}}{\partial x}+v_{2} \frac{\partial h_{2}}{\partial y}\right)+h_{2}\left(\frac{\partial u_{2}}{\partial t}+u_{2} \frac{\partial u_{2}}{\partial x}+v_{2} \frac{\partial u_{2}}{\partial y}\right)+h_{2} u_{2}\left(\frac{\partial u_{2}}{\partial x}+\frac{\partial v_{2}}{\partial y}\right)+ \\
& g h_{2}\left(\frac{\partial h_{1}}{\partial x}+\frac{\partial h_{2}}{\partial x}\right)+2 h_{1} g \frac{\partial h_{2}}{\partial x}=-g h_{2} \frac{\partial \xi}{\partial x}+f v_{2}
\end{aligned}
$$

Using (15) in (21), we obtain

$h_{2} \frac{d u_{2}}{d t}+h_{2} g\left(\frac{\partial h_{1}}{\partial x}+\frac{\partial h_{2}}{\partial x}\right)+2 h_{1} g \frac{\partial h_{2}}{\partial x}=-h_{2} g \frac{\partial \xi}{\partial x}+f v_{2}$

Equation $(e)$ in (13) can be written as:

$$
\begin{aligned}
& v_{2}\left(\frac{\partial h_{2}}{\partial t}+u_{2} \frac{\partial h_{2}}{\partial x}+v_{2} \frac{\partial h_{2}}{\partial y}\right)+h_{2}\left(\frac{\partial v_{2}}{\partial t}+u_{2} \frac{\partial v_{2}}{\partial x}+v_{2} \frac{\partial v_{2}}{\partial y}\right)+h_{2} v_{2}\left(\frac{\partial u_{2}}{\partial x}+\frac{\partial v_{2}}{\partial y}\right)+ \\
& g h_{2}\left(\frac{\partial h_{1}}{\partial y}+\frac{\partial h_{2}}{\partial y}\right)+2 h_{1} g \frac{\partial h_{2}}{\partial y}=-g h_{2} \frac{\partial \xi}{\partial y}-f u_{2}
\end{aligned}
$$

Using (15) in (23), we obtain

$h_{2} \frac{d v_{2}}{d t}+h_{2} g\left(\frac{\partial h_{1}}{\partial y}+\frac{\partial h_{2}}{\partial y}\right)+2 h_{1} g \frac{\partial h_{2}}{\partial y}=-h_{2} g \frac{\partial \xi}{\partial y}-f u_{2}$

Now let $0<f<<1, \quad g=a f$

Suppose the solution $(u, v, h)$ can be expressed in series form as:

$$
\left.\begin{array}{l}
u_{1}(x, y, t)=u_{10}(x, y, t)+f u_{11}(x, y, t)+\ldots \\
u_{2}(x, y, t)=u_{20}(x, y, t)+f u_{21}(x, y, t)+\ldots \\
v_{1}(x, y, t)=v_{10}(x, y, t)+f v_{11}(x, y, t)+\ldots \\
v_{2}(x, y, t)=v_{20}(x, y, t)+f v_{21}(x, y, t)+\ldots \\
h_{1}(x, y, t)=h_{10}(x, y, t)+f h_{11}(x, y, t)+\ldots \\
h_{2}(x, y, t)=h_{20}(x, y, t)+f h_{21}(x, y, t)+\ldots
\end{array}\right\}
$$

Substitute (25) into (15),(16), (18), (20), (22) and (24) and compare the powers of $f$.

Now substituting (25) into (15) yields:

$$
\frac{d h_{10}}{d t}+f \frac{d h_{11}}{d t}+\cdots=-\left(h_{10} \frac{\partial u_{10}}{\partial x}+f h_{10} \frac{\partial u_{11}}{\partial x}+\ldots+f h_{11} \frac{\partial u_{10}}{\partial x}+f^{2} h_{11} \frac{\partial u_{11}}{\partial x}+\cdots\right)
$$

Substituting (25) into (16), we have,

$$
\frac{d h_{20}}{d t}+f \frac{d h_{21}}{d t}+\cdots=-\left(h_{20} \frac{\partial u_{20}}{\partial x}+f h_{20} \frac{\partial u_{2}}{\partial x}+\cdots+f h_{21} \frac{\partial u_{20}}{\partial x}+f^{2} h_{21} \frac{\partial u_{21}}{\partial x}+\cdots\right)
$$

Substituting (25) into (18), we have, 
$\left.\begin{array}{l}h_{10} \frac{d u_{10}}{d t}+f h_{10} \frac{d u_{11}}{d t}+f h_{11} \frac{d u_{10}}{d t}+f^{2} h_{11} \frac{d u_{11}}{d t}+\ldots+ \\ a f\left(\begin{array}{l}h_{10} \frac{\partial h_{10}}{\partial x}+h_{10} f \frac{\partial h_{11}}{\partial x}+f h_{11} \frac{\partial h_{10}}{\partial x}+f^{2} h_{11} \frac{\partial h_{11}}{\partial x} \ldots+ \\ h_{10} \frac{\partial h_{20}}{\partial x}+f h_{10} \frac{\partial h_{21}}{\partial x}+f h_{11} \frac{\partial}{\partial x}+f^{2} h_{11} \frac{\partial h_{21}}{\partial x}\end{array}\right)= \\ a f\left(h_{10} \frac{\partial \xi}{\partial x}+f h_{11} \frac{\partial \xi}{\partial x}+\ldots\right)+f\left(v_{10}+f v_{11}+\ldots\right)\end{array}\right\}$

Substituting (25) into (20), we have,

$$
\left.\begin{array}{l}
h_{10} \frac{d v_{10}}{d t}+f h_{10} \frac{d v_{11}}{d t}+f h_{11} \frac{d v_{10}}{d t}+f^{2} h_{11} \frac{d v_{11}}{d t}+\ldots+ \\
a f\left(\begin{array}{l}
h_{10} \frac{\partial h_{10}}{\partial y}+h_{10} f \frac{\partial h_{11}}{\partial y}+f h_{11} \frac{\partial h_{10}}{\partial y}+f^{2} h_{11} \frac{\partial h_{11}}{\partial y} \ldots+ \\
h_{10} \frac{\partial h_{20}}{\partial y}+f h_{10} \frac{\partial h_{21}}{\partial y}+f h_{11} \frac{\partial}{\partial y}+f^{2} h_{11} \frac{\partial h_{21}}{\partial y x}
\end{array}\right)= \\
-a f\left(h_{10} \frac{\partial \xi}{\partial y}+f h_{11} \frac{\partial \xi}{\partial y}+\ldots\right)-f\left(u_{10}+f u_{11}\right)
\end{array}\right)
$$

Substituting (25) into (22), we have,

$$
\left.\begin{array}{l}
\left(h_{20} \frac{d u_{20}}{d t}+f h_{20} \frac{d u_{22}}{d t}+f h_{21} \frac{d u_{20}}{d t}+f^{2} h_{21} \frac{d u_{21}}{d t}+\cdots\right)+ \\
a f\left(\begin{array}{l}
h_{20} \frac{\partial h_{10}}{\partial x}+f h_{20} \frac{\partial h_{10}}{\partial x}+f h_{21} \frac{\partial h_{10}}{\partial x}+f^{2} h_{21} \frac{\partial h_{10}}{\partial x} \cdots+h_{20} \frac{\partial h_{20}}{\partial x}+f h_{20} \frac{\partial h_{20}}{\partial x} \\
+f h_{21} \frac{\partial h_{21}}{\partial x}+f^{2} h_{21} \frac{\partial h_{21}}{\partial x}+\cdots
\end{array}\right)+ \\
2 a f\left(h_{10} \frac{\partial h_{20}}{\partial x}+f h_{11} \frac{\partial h_{20}}{\partial x}+h_{10} \frac{\partial f h_{21}}{\partial x}+f^{2} h_{11} \frac{\partial f h_{21}}{\partial x}+\cdots\right)= \\
-a f\left(h_{20} \frac{\partial \xi}{\partial x}+f h_{21} \frac{\partial \xi}{\partial x}+\ldots\right)+f\left(v_{20}+f v_{21}\right)+\cdots
\end{array}\right\}
$$

Substituting (31) into (30), we have, 


$$
\left.\begin{array}{l}
\left(h_{20} \frac{d v_{20}}{d t}+f h_{20} \frac{d v_{21}}{d t}+f h_{21} \frac{d v_{20}}{d t}+f^{2} h_{21} \frac{d v_{21}}{d t}+\cdots\right)+ \\
a f\left(\begin{array}{c}
h_{20} \frac{\partial h_{10}}{\partial x}+f h_{20} \frac{\partial h_{10}}{\partial x}+f h_{21} \frac{\partial h_{10}}{\partial x}+f^{2} h_{21} \frac{\partial h_{10}}{\partial x}+\cdots \\
\left.+h_{20} \frac{\partial h_{20}}{\partial x}+f h_{20} \frac{\partial h_{20}}{\partial x}+f h_{21} \frac{\partial h_{21}}{\partial x}+f^{2} h_{21} \frac{\partial h_{21}}{\partial x}+\cdots\right)
\end{array}\right)+ \\
2 a f\left(h_{10} \frac{\partial h_{20}}{\partial x}+f h_{11} \frac{\partial h_{20}}{\partial y}+h_{10} \frac{\partial f h_{21}}{\partial y}+f^{2} h_{11} \frac{\partial f h_{21}}{\partial y}+\cdots\right)= \\
-a f\left(h_{20} \frac{\partial \xi}{\partial y}+f h_{21} \frac{\partial \xi}{\partial y}+\ldots\right)-f\left(u_{20}+u_{21}+\cdots\right)
\end{array}\right\}
$$

Thus comparing the coefficients of the powers of $f$, we have;

$f^{0}:$

$$
h_{10} \frac{d u_{10}}{d t}=0 \quad ; \quad u_{10}(x, y, 0)=u_{0}
$$

$$
h_{10} \frac{d v_{10}}{d t}=0 \quad ; \quad v_{10}(x, y, 0)=v_{0}
$$

$h_{20} \frac{d u_{20}}{d t}=0 \quad ; \quad u_{20}(x, y, 0)=u_{0}$

$h_{20} \frac{d v_{20}}{d t}=0 \quad ; \quad v_{20}(x, y, 0)=v_{0}$

$$
\frac{d h_{10}}{d t}=-h_{10}\left(\frac{\partial u_{10}}{\partial x}+\frac{\partial v_{10}}{\partial y}\right)
$$

$\frac{d h_{20}}{d t}=-h_{20}\left(\frac{\partial u_{20}}{\partial x}+\frac{\partial v_{20}}{\partial y}\right)$

$f^{1}:$

$h_{10} \frac{d u_{11}}{d t}+h_{11} \frac{d u_{10}}{d t}+a h_{10}\left(\frac{\partial h_{10}}{\partial x}+\frac{\partial h_{20}}{\partial x}\right)=-a h_{10} \frac{\partial \xi}{\partial x}+v_{10}$

$h_{10} \frac{d v_{11}}{d t}+h_{11} \frac{d v_{10}}{d t}+a h_{10}\left(\frac{\partial h_{10}}{\partial y}+\frac{\partial h_{20}}{\partial y}\right)=-a h_{10} \frac{\partial \xi}{\partial y}-u_{10}$

$h_{20} \frac{d u_{21}}{d t}+h_{21} \frac{d u_{20}}{d t}+a h_{20}\left(\frac{\partial h_{10}}{\partial x}+\frac{\partial h_{20}}{\partial x}\right)+2 h_{10} a \frac{\partial h_{20}}{\partial x}=-a h_{20} \frac{\partial \xi}{\partial x}+v_{20}$

$$
h_{20} \frac{d v_{21}}{d t}+h_{21} \frac{d v_{20}}{d t}+a h_{20}\left(\frac{\partial h_{10}}{\partial y}+\frac{\partial h_{20}}{\partial y}\right)+2 h_{10} a \frac{\partial h_{20}}{\partial y}=-a h_{20} \frac{\partial \xi}{\partial y}-u_{20}
$$




$$
\begin{aligned}
& \frac{d h_{11}}{d t}=-h_{11}\left(\frac{\partial u_{10}}{\partial x}+\frac{\partial v_{10}}{\partial y}\right)-h_{10}\left(\frac{\partial u_{11}}{\partial x}+\frac{\partial v_{11}}{\partial y}\right) \\
& \frac{d h_{21}}{d t}=-h_{21}\left(\frac{\partial u_{20}}{\partial x}+\frac{\partial v_{20}}{\partial y}\right)-h_{20}\left(\frac{\partial u_{21}}{\partial x}+\frac{\partial v_{21}}{\partial y}\right)
\end{aligned}
$$

Integrating (32) with respect to $t$, we have;

(44)

Similarly, Integrating $(33),(34)$, and (35)with respect to $t$ we have;

$$
\begin{aligned}
& v_{10}(x, y, t)=v_{0} \\
& u_{20}(x, y, t)=u_{0} \\
& v_{20}(x, y, t)=v_{0}
\end{aligned}
$$

Hence, equation (36) reduces to;

$$
\frac{d h_{10}}{d t}=0 \quad, h_{10}(x, y, 0)=m e^{-s\left(x^{2}+y^{2}\right)}-\alpha \sin \beta x
$$

Integrating this with respect to $t$, we have;

$h_{10}(x, y, t)=m e^{-s\left(x^{2}+y^{2}\right)}-\alpha \sin \beta x$

Similarly, equation (43) reduces to;

$$
\frac{d h_{20}}{d t}=0 \quad, \quad h_{20}(x, y, 0)=1-\alpha \sin \beta x
$$

So that integrating this with respect to $t$, we have;

$h_{20}(x, y, t)=1-\alpha \sin \beta x$

Also, equation (38) reduces to;

$$
\begin{aligned}
& h_{10} \frac{d u_{11}}{d t}=-a h_{10}\left(\frac{\partial h_{10}}{\partial x}+\frac{\partial h_{20}}{\partial x}\right)-a h_{10} \frac{\partial \xi}{\partial x}+v_{10}, \quad h_{10}=m e^{-s\left(x^{2}+y^{2}\right)}-\alpha \sin \beta x \\
& \frac{d u_{11}}{d t}=-a \frac{\partial h_{10}}{\partial x}-a \frac{\partial h_{20}}{\partial x}-a \frac{\partial \xi}{\partial x}+\frac{v_{10}}{h_{10}} \\
& \frac{d u_{11}}{d t}=2 a m s x e^{-s\left(x^{2}+y^{2}\right)}+a \alpha \beta \cos \beta x+\frac{v_{0}}{m e^{-s\left(x^{2}+y^{2}\right)}-\alpha \sin \beta x}
\end{aligned}
$$

Integrating this with respect to $t$, we have;

$$
u_{11}(x, y, t)=\left(2 a m s x e^{-s\left(x^{2}+y^{2}\right)}+a \alpha \beta \cos \beta x+\frac{v_{0}}{m e^{-s\left(x^{2}+y^{2}\right)}-\alpha \sin \beta x}\right) t
$$


Equation (39) reduces to;

$$
\begin{aligned}
& \frac{d v_{11}}{d t}=-a\left(\frac{\partial h_{10}}{\partial y}+\frac{\partial h_{20}}{\partial y}\right)-a \frac{\partial \xi}{\partial y}-\frac{u_{10}}{h_{10}} \\
& \frac{d v_{11}}{d t}=-a \frac{\partial h_{10}}{\partial y}-a \frac{\partial \xi}{\partial y}-\frac{u_{10}}{h_{10}}=2 a m s y e^{-s\left(x^{2}+y^{2}\right)}-\frac{u_{0}}{m e^{-s\left(x^{2}+y^{2}\right)}-\alpha \sin \beta x}
\end{aligned}
$$

Integrating this with respect to $t$, we have;

$$
v_{11}(x, y, t)=\left(2 a m s y e^{-s\left(x^{2}+y^{2}\right)}-\frac{u_{0}}{m e^{-s\left(x^{2}+y^{2}\right)}-\alpha \sin \beta x}\right) t
$$

Equation (40) reduces to;

$$
\begin{aligned}
& h_{20} \frac{d u_{21}}{d t}+a h_{20}\left(\frac{\partial h_{10}}{\partial x}+\frac{\partial h_{20}}{\partial x}\right)+2 h_{10} a \frac{\partial h_{20}}{\partial x}=-a h_{20} \frac{\partial \xi}{\partial x}+v_{20} \\
\frac{d u_{21}}{d t}=- & -a\left(\frac{\partial h_{10}}{\partial x}+\frac{\partial h_{20}}{\partial x}\right)-\frac{2 a h_{10}}{h_{20}} \frac{\partial h_{20}}{\partial x}-a \frac{\partial \xi}{\partial x}+\frac{v_{20}}{h_{20}} \\
= & 2 a m s x e^{-s\left(x^{2}+y^{2}\right)}+a \alpha \beta \cos \beta x+\frac{2 a m \alpha \beta e^{-s\left(x^{2}+y^{2}\right)} \cos \beta x}{1-\alpha \sin \beta x}-\frac{2 a \alpha^{2} \beta \sin \beta x \cos \beta x}{1-\alpha \sin \beta x}+\frac{v_{0}}{1-\alpha \sin \beta x}
\end{aligned}
$$

Integrating this with respect to $t$, we have;

$$
u_{21}=\left(\begin{array}{l}
2 a m s x e^{-s\left(x^{2}+y^{2}\right)}+a \alpha \beta \cos \beta x+\frac{2 a m \alpha \beta e^{-s\left(x^{2}+y^{2}\right)} \cos \beta x}{1-\alpha \sin \beta x}- \\
\frac{2 a \alpha^{2} \beta \sin \beta x \cos \beta x}{1-\alpha \sin \beta x}+\frac{v_{0}}{1-\alpha \sin \beta x}
\end{array}\right) t
$$

Equation (41) reduces to;

That is

$$
\frac{d v_{21}}{d t}=-a \frac{\partial h_{10}}{\partial y}-\frac{u_{0}}{h_{20}}=2 a m s y e^{-s\left(x^{2}+y^{2}\right)}-\frac{u_{0}}{1-\alpha \sin \beta x}
$$

$v_{21}(x, y, t)=\left(2 a m s y e^{-s\left(x^{2}+y^{2}\right)}-\frac{u_{0}}{1-\alpha \sin \beta x}\right) t$

Equation (42) reduces to; $\frac{d h_{11}}{d t}=-h_{10}\left(\frac{\partial u_{11}}{\partial x}+\frac{\partial v_{11}}{\partial y}\right)$ which implies that

$$
\begin{aligned}
& \frac{\partial u_{11}}{\partial x}=\left(2 a m s e^{-s\left(x^{2}+y^{2}\right)}-4 a m s^{2} x^{2} e^{-s\left(x^{2}+y^{2}\right)}-a \alpha \beta^{2} \sin (\beta x)+\frac{v_{0}\left(2 m s x e^{-s\left(x^{2}+y^{2}\right)}+\alpha \beta \cos (\beta x)\right)}{\left(m e^{-s\left(x^{2}+y^{2}\right)}-\alpha \sin (\beta x)\right)^{2}}\right) t . \\
& \frac{\partial v_{11}}{\partial y}=\left(2 a m s e^{-5\left(x^{2}+y^{2}\right)}-4 a m s^{2} y^{2} e^{-5\left(x^{2}+y^{2}\right)}-\frac{2 u_{0} m s y e^{-s\left(x^{2}+y^{2}\right)}}{\left(m e^{-s\left(x^{2}+y^{2}\right)}-\alpha \sin (\beta x)\right)^{2}}\right) t .
\end{aligned}
$$

Therefore; 


$$
\frac{\partial h_{11}}{\partial t}=\left(\alpha \sin \beta x-m e^{\left.-s\left(x^{2}+y^{2}\right)\right)}\left(\begin{array}{l}
4 a m s e^{-s\left(x^{2}+y^{2}\right)}-4 a m s^{2} x^{2} e^{-s\left(x^{2}+y^{2}\right)}-a \alpha \beta^{2} \sin (\beta x)+ \\
\frac{v_{0}\left(2 m s x e^{-s\left(x^{2}+y^{2}\right)}+\alpha \beta \cos (\beta x)\right)}{\left(m e^{-s\left(x^{2}+y^{2}\right)}-\alpha \sin (\beta x)\right)^{2}}-4 a m s^{2} y^{2} e^{-s\left(x^{2}+y^{2}\right)}-\frac{2 u_{0} m s y e^{-s\left(x^{2}+y^{2}\right)}}{\left(m e^{-s\left(x^{2}+y^{2}\right)}-\alpha \sin (\beta x)\right)^{2}}
\end{array}\right) t .\right.
$$

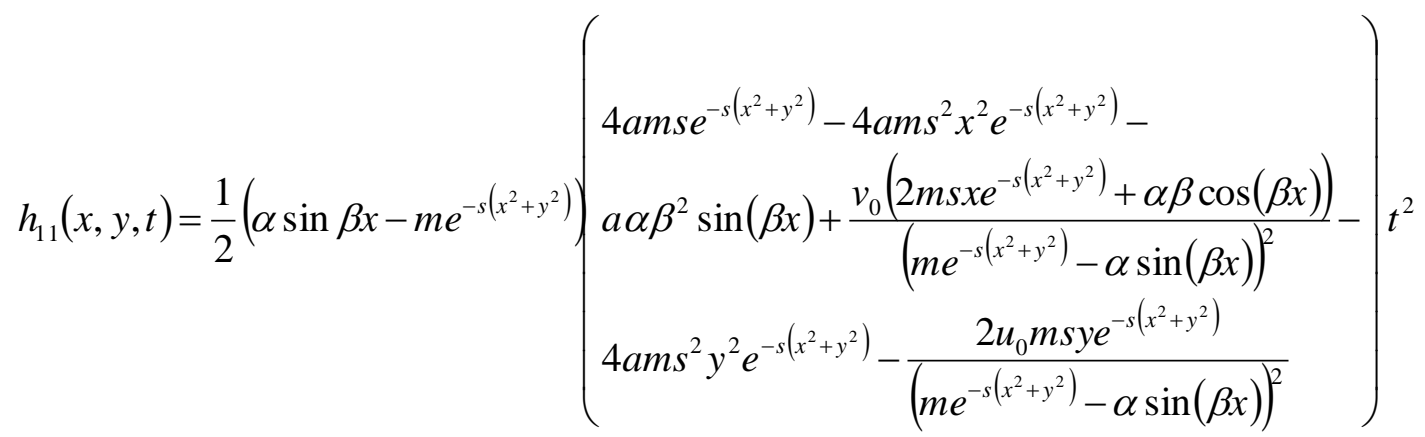

Equation (43) reduces to;

$$
\begin{aligned}
& \frac{d h_{21}}{d t}=-h_{o}\left(\frac{\partial u_{21}}{\partial x}+\frac{\partial v_{21}}{\partial y}\right) \quad \text { which implies that } \\
& \frac{\partial u_{21}}{\partial x}=\left(\begin{array}{l}
2 a m s e^{-s\left(x^{2}+y^{2}\right)}-4 a m s^{2} x^{2} e^{-s\left(x^{2}+y^{2}\right)}-a \alpha \beta^{2} \sin (\beta x)-\frac{4 a m s x \alpha \beta \cos (\beta x) e^{-s\left(x^{2}+y^{2}\right)}}{1-\alpha \sin (\beta x)}- \\
\frac{2 a m \alpha \beta^{2} \sin (\beta x) e^{-s\left(x^{2}+y^{2}\right)}}{1-\alpha \sin (\beta x)}+\frac{2 a m \alpha^{2} \beta^{2} \cos ^{2}(\beta x) e^{-s\left(x^{2}+y^{2}\right)}}{(1-\alpha \sin (\beta x))^{2}}+\frac{2 a \alpha^{2} \beta^{2} \sin ^{2}(\beta x)}{1-\alpha \sin (\beta x)}-\frac{a \alpha^{2} \beta^{2} \cos ^{2}(\beta x)}{1-\alpha \sin (\beta x)}- \\
\frac{2 a \alpha^{3} \beta^{2} \cos ^{2}(\beta x) \sin (\beta x)}{(1-\alpha \sin (\beta x))^{2}}+\frac{v_{0} \alpha \beta \cos (\beta x)}{(1-\alpha \sin (\beta x))^{2}}
\end{array}\right) t .
\end{aligned}
$$$$
\frac{\partial v_{21}}{\partial y}=\left(2 a m s e^{-s\left(x^{2}+y^{2}\right)}-4 a m s^{2} y^{2} e^{-5\left(x^{2}+y^{2}\right)}\right) t \text { and }
$$

$$
\begin{aligned}
\frac{\partial h_{21}}{\partial t}= & (\alpha \sin (\beta x)-1) \times \\
& \left(\begin{array}{l}
4 a m s e^{-s\left(x^{2}+y^{2}\right)}-4 a m s^{2} x^{2} e^{-s\left(x^{2}+y^{2}\right)}-4 a m s^{2} y^{2} e^{-s\left(x^{2}+y^{2}\right)}-a \alpha \beta^{2} \sin (\beta x)-\frac{4 a m s x \alpha \beta \cos (\beta x) e^{-s\left(x^{2}+y^{2}\right)}}{1-\alpha \sin (\beta x)}- \\
\frac{2 a m \alpha \beta^{2} \sin (\beta x) e^{-s\left(x^{2}+y^{2}\right)}}{1-\alpha \sin (\beta x)}+\frac{2 a m \alpha^{2} \beta^{2} \cos ^{2}(\beta x) e^{-s\left(x^{2}+y^{2}\right)}}{(1-\alpha \sin (\beta x))^{2}}+\frac{2 a \alpha^{2} \beta^{2} \sin ^{2}(\beta x)}{1-\alpha \sin (\beta x)}-\frac{a \alpha^{2} \beta^{2} \cos ^{2}(\beta x)}{1-\alpha \sin (\beta x)}- \\
\frac{2 a \alpha^{3} \beta^{2} \cos ^{2}(\beta x) \sin (\beta x)}{(1-\alpha \sin (\beta x))^{2}}+\frac{v_{0} \alpha \beta \cos (\beta x)}{(1-\alpha \sin (\beta x))^{2}}
\end{array}\right) t
\end{aligned}
$$

Thus;

$$
\begin{aligned}
& h_{21}(x, y, t)=\frac{1}{2}(\alpha \sin (\beta x)-1) \times \\
& \left(\begin{array}{l}
4 a m s e^{-s\left(x^{2}+y^{2}\right)}-4 a m s^{2} x^{2} e^{-s\left(x^{2}+y^{2}\right)}-4 a m s^{2} y^{2} e^{-s\left(x^{2}+y^{2}\right)}-a \alpha \beta^{2} \sin (\beta x)- \\
\frac{4 a m s x \alpha \beta \cos (\beta x) e^{-s\left(x^{2}+y^{2}\right)}}{1-\alpha \sin (\beta x)}-\frac{2 a m \alpha \beta^{2} \sin (\beta x) e^{-s\left(x^{2}+y^{2}\right)}}{1-\alpha \sin (\beta x)}+\frac{2 a m \alpha^{2} \beta^{2} \cos ^{2}(\beta x) e^{-s\left(x^{2}+y^{2}\right)}}{(1-\alpha \sin (\beta x))^{2}}+t^{2} \\
\frac{2 a \alpha^{2} \beta^{2} \sin ^{2}(\beta x)}{1-\alpha \sin (\beta x)}-\frac{a \alpha^{2} \beta^{2} \cos ^{2}(\beta x)}{1-\alpha \sin (\beta x)}-\frac{2 a \alpha^{3} \beta^{2} \cos ^{2}(\beta x) \sin (\beta x)}{(1-\alpha \sin (\beta x))^{2}}+\frac{v_{0} \alpha \beta \cos (\beta x)}{(1-\alpha \sin (\beta x))^{2}}
\end{array}\right)
\end{aligned}
$$


Therefore; from equation (25)

$$
\begin{aligned}
& u_{1}(x, y, t)=u_{10}(x, y, t)+f u_{11}(x, y, t)+\ldots \\
& u_{1}(x, y, t)=u_{0}+\left(2 g m s x e^{-s\left(x^{2}+y^{2}\right)}+g \alpha \beta \cos (\beta x)-\frac{f v_{0}}{m e^{-s\left(x^{2}+y^{2}\right)}-\alpha \sin (\beta x)}\right) t \\
& u_{2}(x, y, t)=u_{20}(x, y, t)+f u_{21}(x, y, t)+\cdots \\
& v_{1}(x, y, t)=v_{10}(x, y, t)+f v_{11}(x, y, t)+\cdots \\
& u_{2}(x, y, t)=u_{0}+\left(\begin{array}{l}
2 g m s x e^{-s\left(x^{2}+y^{2}\right)}+g \alpha \beta \cos (\beta x)+\frac{2 g m \alpha \beta \cos (\beta x) e^{-s\left(x^{2}+y^{2}\right)}}{(1-\alpha \sin (\beta x))}- \\
\frac{2 g \alpha^{2} \beta \cos (\beta x) \sin (\beta x)}{(1-\alpha \sin (\beta x))}+\frac{f v_{0}}{1-\alpha \sin (\beta x)}
\end{array}\right) t \\
& v_{1}(x, y, t)=v_{0}+\left(2 g m s y e^{-s\left(x^{2}+y^{2}\right)}-\frac{f u_{0}}{m e^{-5\left(x^{2}+y^{2}\right)}-\alpha \sin (\beta x)}\right) t \\
& v_{2}(x, y, t)=v_{20}(x, y, t)+f v_{21}(x, y, t)+\cdots \\
& v_{2}(x, y, t)=v_{0}+\left(2 g m s y e^{-s\left(x^{2}+y\right)}-\frac{f u_{0}}{1-\alpha \sin (\beta x)}\right) t
\end{aligned}
$$

Therefore,

$$
h_{1}(x, y, t)=h_{10}(x, y, t)+f h_{11}(x, y, t)+\cdots
$$

which implies that,

$$
\left.\begin{array}{l}
h_{1}(x, y, t)=m e^{-s\left(x^{2}+y^{2}\right)}-\alpha \sin (\beta x)+\frac{1}{2}\left(\alpha \sin (\beta x)-m e^{-s\left(x^{2}+y^{2}\right)}\right) \times \\
\left(\begin{array}{l}
4 g m s e^{-s\left(x^{2}+y^{2}\right)}-4 g m s^{2} x^{2} e^{-s\left(x^{2}+y^{2}\right)}-4 g m s^{2} y^{2} e^{-s\left(x^{2}+y^{2}\right)}-g s \alpha \beta^{2} \sin (\beta x)+ \\
\frac{f v_{0}\left(2 m s x e^{-s\left(x^{2}+y^{2}\right)}-\alpha \beta \cos (\beta x)\right)}{\left(m e^{-s\left(x^{2}+y^{2}\right)}-\alpha \sin (\beta x)\right)^{2}}-\frac{2 m s y u_{0} e^{-s\left(x^{2}+y^{2}\right)}}{\left(m e^{-s\left(x^{2}+y^{2}\right)}-\beta \sin (\beta x)\right)^{2}}
\end{array}\right) t^{2}
\end{array}\right\}
$$

Similarly,

$$
h_{2}(x, y, t)=h_{20}(x, y, t)+f h_{21}(x, y, t)+\cdots
$$

which implies that 


$$
\left.\begin{array}{l}
h_{2}(x, y, t)=1-\alpha \sin (\beta x)+\frac{1}{2}(\alpha \sin (\beta x)-1) \times \\
\left.\begin{array}{l}
4 g m s e^{-s\left(x^{2}+y^{2}\right)}-4 g m s^{2} x^{2} e^{-s\left(x^{2}+y^{2}\right)}-4 g m s^{2} y^{2} e^{-s\left(x^{2}+y^{2}\right)}-g \alpha \beta^{2} \sin (\beta x) \\
-\frac{4 g m s x \alpha \beta \cos (\beta x) e^{-s\left(x^{2}+y^{2}\right)}}{(1-\alpha \sin (\beta x))}-\frac{2 g m x \alpha \beta^{2} \sin (\beta x) e^{-5\left(x^{2}+y^{2}\right)}}{1-\alpha \sin (\beta x)} \\
+\frac{2 g m \alpha^{2} \beta^{2} \cos ^{2}(\beta x) e^{-s\left(x^{2}+y^{2}\right)}}{(1-\alpha \sin (\beta x))^{2}}+\frac{2 g \alpha^{2} \beta^{2} \sin ^{2}(\beta x)}{1-\alpha \sin (\beta x)}-\frac{2 g \alpha^{2} \beta^{2} \cos (\beta x)}{1-\alpha \sin (\beta x)} \\
-\frac{2 g \alpha^{3} \beta^{2} \cos ^{2}(\beta x) \sin (\beta x)}{(1-\alpha \sin (\beta x))^{2}}+\frac{v_{0} \alpha \beta \cos (\beta x)}{(1-\alpha \sin (\beta x))^{2}}
\end{array}\right\} t^{2}
\end{array}\right\}
$$

\section{Results and Discussion}

Using Maple software, the following results were obtained graphically to help interpret the result of the modeled equations. Thus;

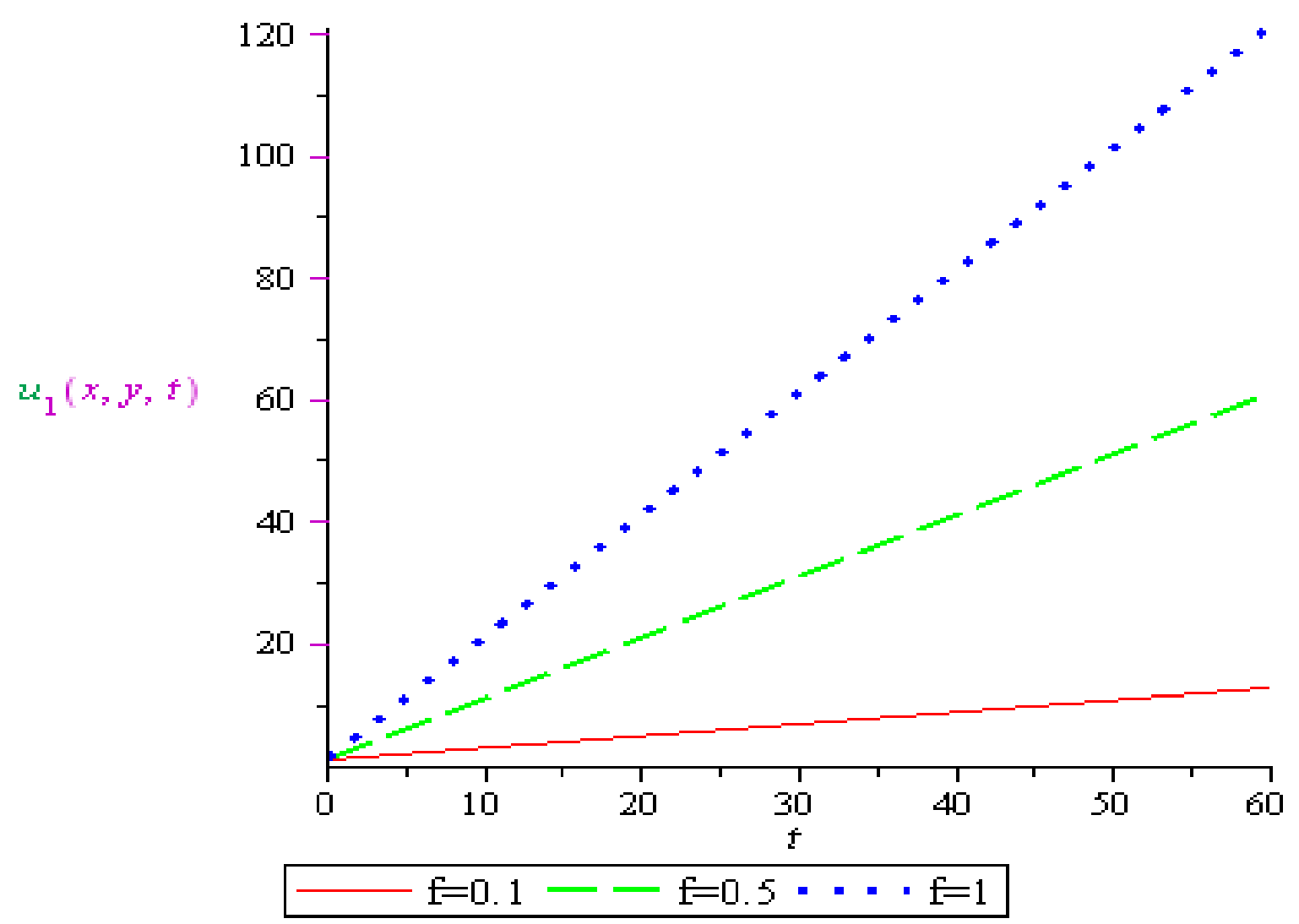

figure 1 variation of velocity $u_{1}(x, y, t)$ with time $t$, with different values of 


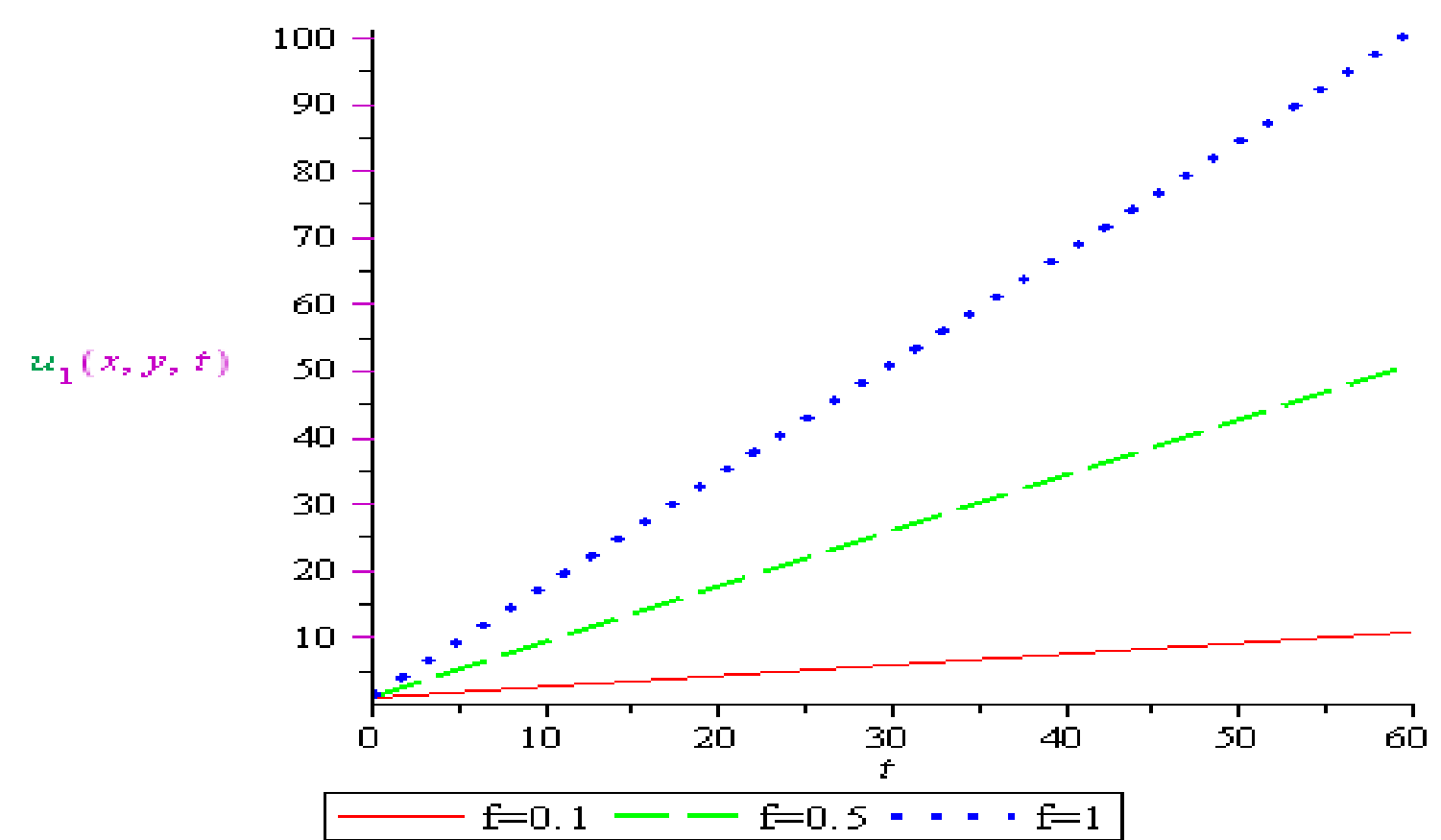

figure 2 variation of velocity $u_{2}(x, y, t)$ with time $t$, with different values of f.

Figure 1 and 2 shows the flow rate in the stratified fluid flow of two layers $u_{1}(x, y, t)$ and $u_{2}(x, y, t)$ where, $u_{1}(x, y, t)$ is the rate of flow in the first layer and $u_{2}(x, y, t)$ in the second layer. At $f=0.1$ the flow velocity is slowly varying but increases gradually with time in first and second layers, although faster in the first layer than in the second. At $f=0.5$ the flow velocity increases rapidly as time increases both in the first and second layers and these appeard faster when compared to $f=0.1$
. At $f=1$ the flow velocity is very high and increases with time and these velocities are greater than what obtained in both $f=0.1$ and $f=0.5$. This shows that the flow rate $u_{2}(x, y, t)$ in the second layer which is denser than that in the first layer is slow as compared to the flow velocity $u_{1}(x, y, t)$. Furthermore, the result shows that in in all cases, the higher the force due to rotation, the faster the rate of flow. 


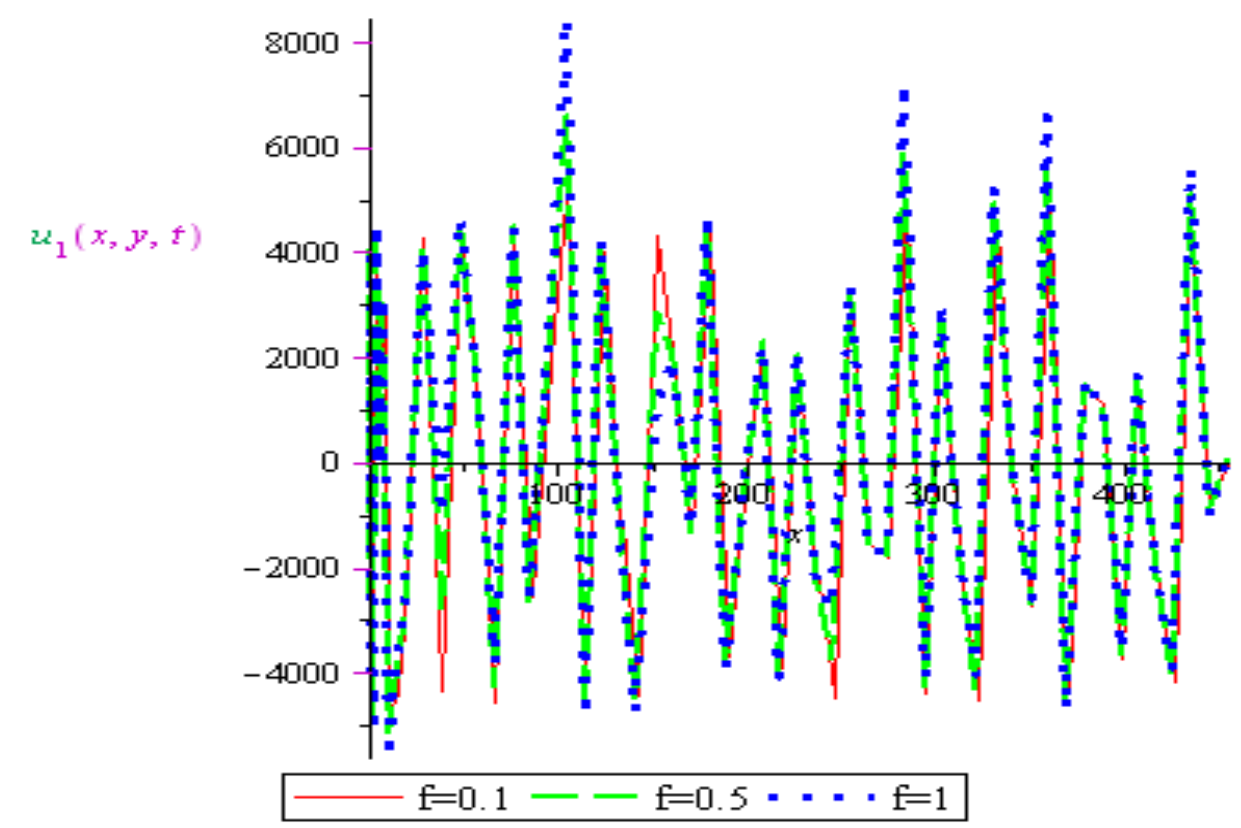

figure 3 variation of velocity $u_{1}(x, y, t)$ with distance $x$, with different values of $f$.

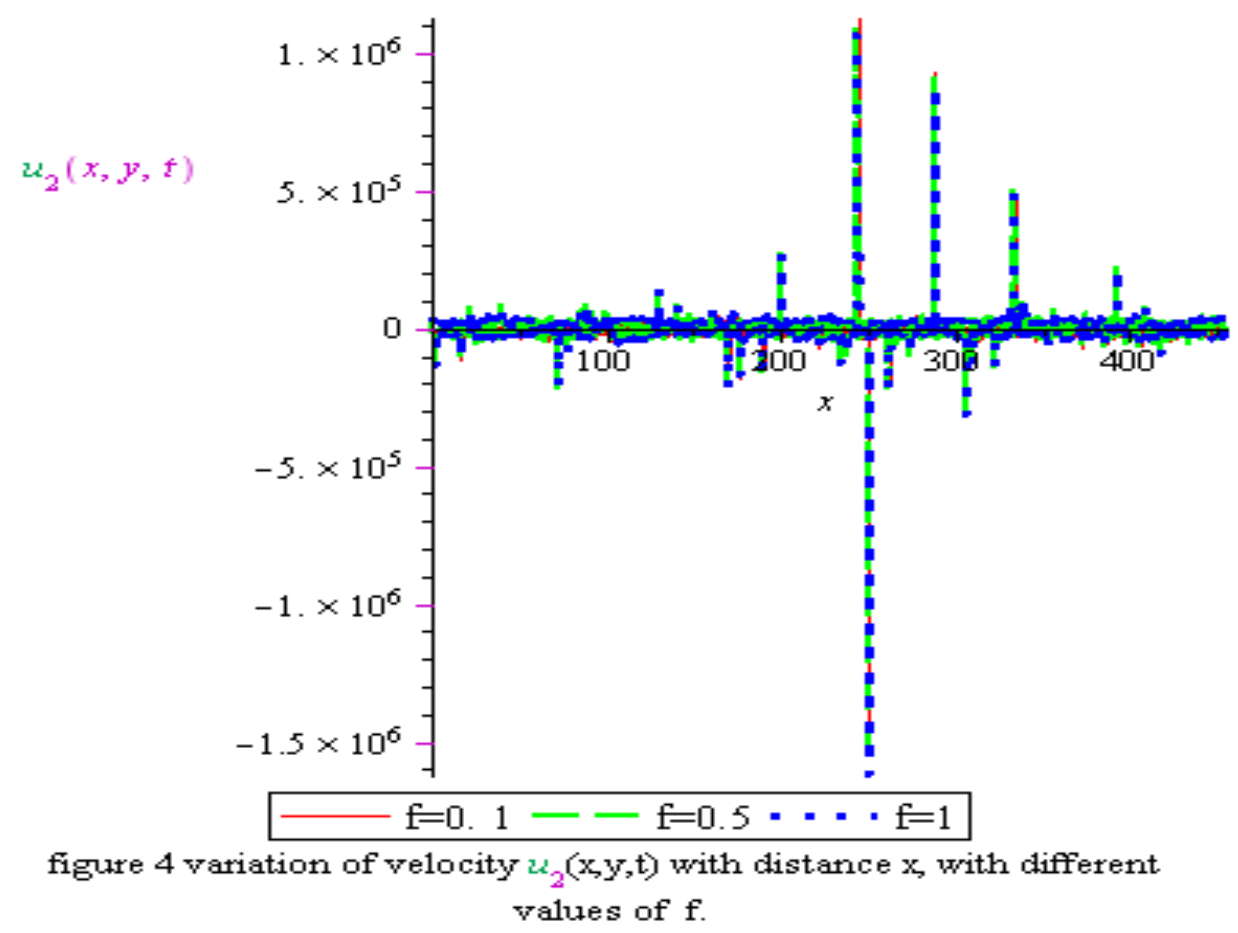

The graphs of figure $3 \& 4$ above shows the flow velocity $u_{1}(x, y, t)$ and $u_{2}(x, y, t)$ in the horizontal $x$-direction in the stratifiedd fluid of two layers. The figures show a flow system characterized by disordered changes in flow velocity. The velocities are varying in space due to turbulent fluctuations and the deviation of the set of haphazard velocity fluctuations with different levels of turbulence. For the top layer, we may note that sunlight, wind and other environmental factors may increase the temperature of the upper layer thereby changing the density of it and thus making the flow faster than that of the lower layer.These correspond to the flow at the free surface of a body of shallow water under the force of gravity, or to the flow below a horizontal pressure surface in a fluid. A surface wave travels at the free surface of a fluid. The maximum velocity of the wave and the maximum displacement of fluid particles occur at the free surface of the fluid. To be noted is 
the fact that there is more congestion in the second layer as there are more obstructions in the dowmward part than in the upper part and the density is denser there than in the upper part . If there is any barrier at the water bed, the effect is felt in the second layer more than in the upper layer. Since the second layer is closer to the bed the rate of randomness and fluctuation is higher there than that at the surface layer. The flow in this layer is known as internal wave which travels within the interior of a fluid. The maximum velocity and maximum amplitude occur within the fluid or at an internal boundary (interface) as internal waves depend on the density-stratification of the fluid.

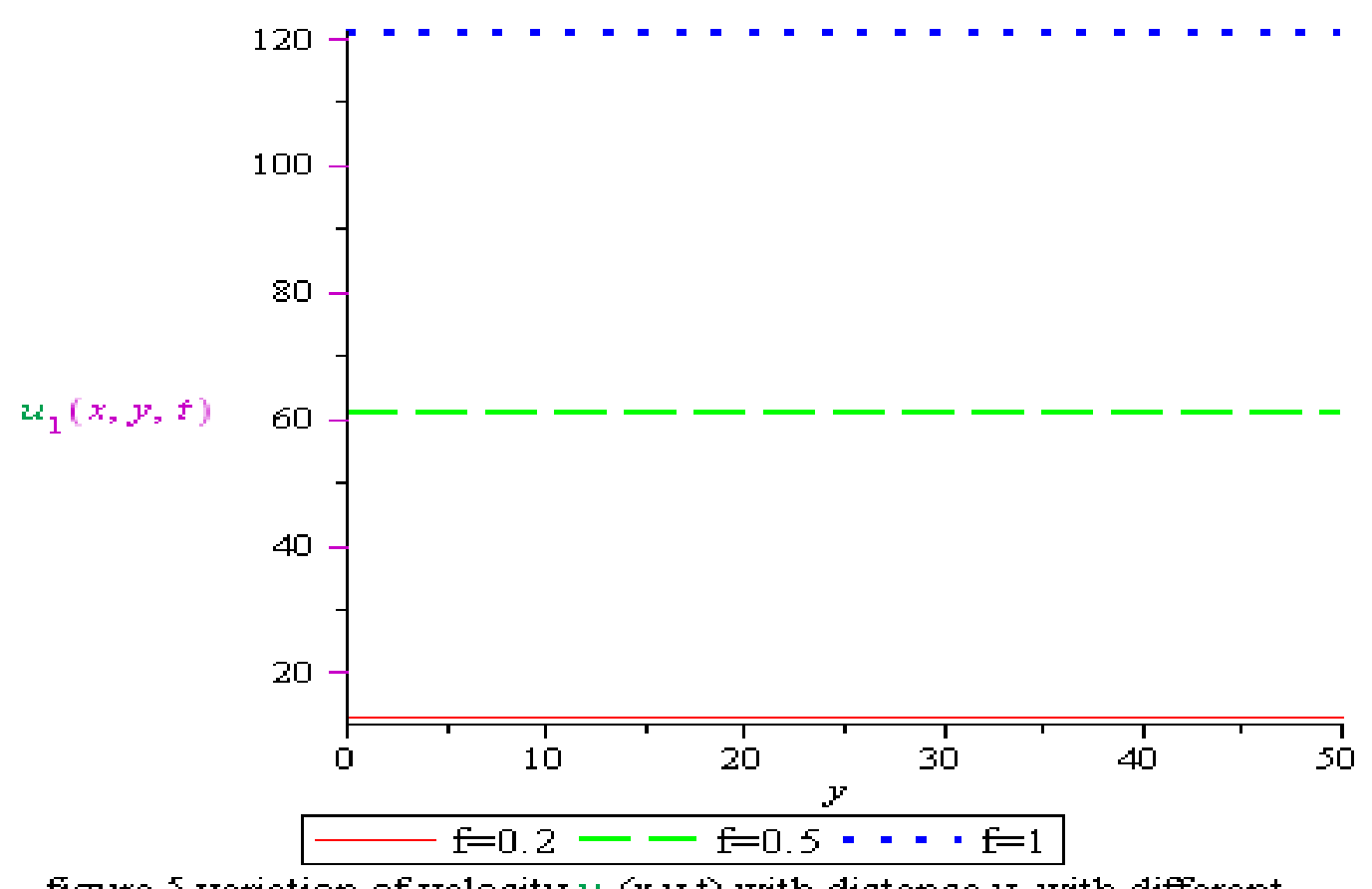

figure 5 variation of velocity $u_{1}(x, y, t)$ with distance $y$, with different

values of $f$.

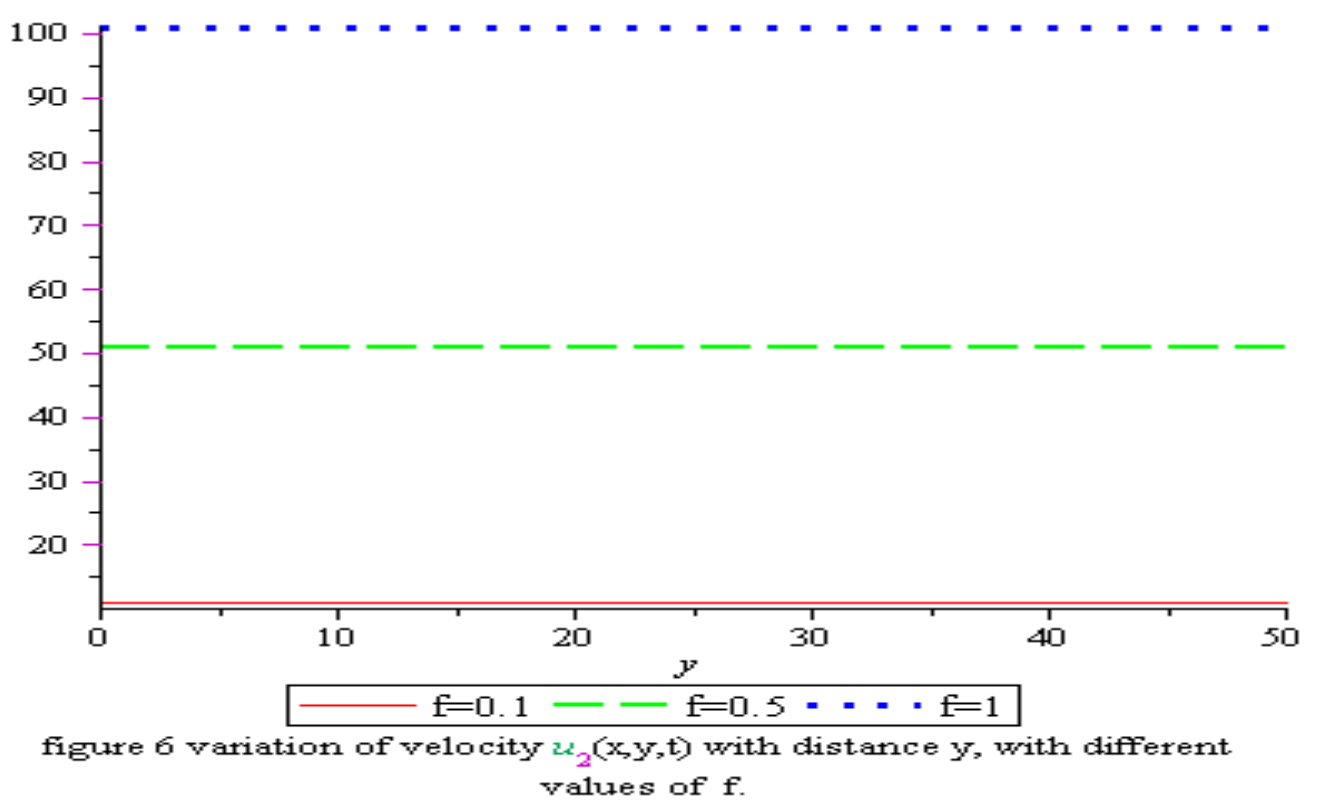


Figure 5 and 6 also illustrates the flow velocity $u_{1}(x, y, t)$ and $u_{2}(x, y, t)$ in the $y$ - direction that has constant motion. At $f=0.1$, the flow is not experinced at all points in $y$-.direction. However, when the force due to rotation is $f=0.5$ and $f=1$ the constant flow velocity of $60 \mathrm{~m} / \mathrm{s}$ and $120 \mathrm{~m} / \mathrm{s}$ were experinced at different points for $u_{1}(x, y, t)$ and for that of $u_{2}(x, y, t)$, the constant flow velocity of $50 \mathrm{~m} / \mathrm{s}$ and $100 \mathrm{~m} / \mathrm{s}$ were also experienced. This shows that, the flow velocity is high on the upper layer than in the down layer

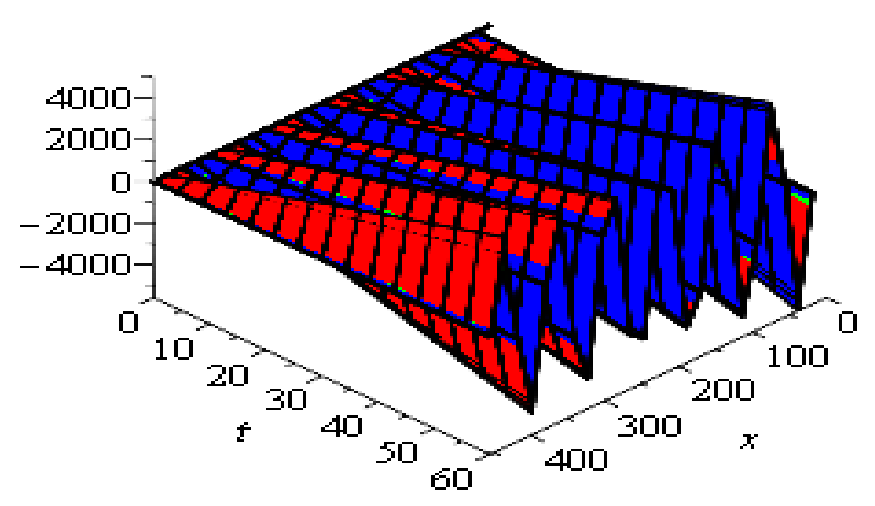

figure 7 : variation of velocity

$u_{1} \mid x, y, t ;$ with the horizontal direction $-x$ and time $t$ for different values of $f$

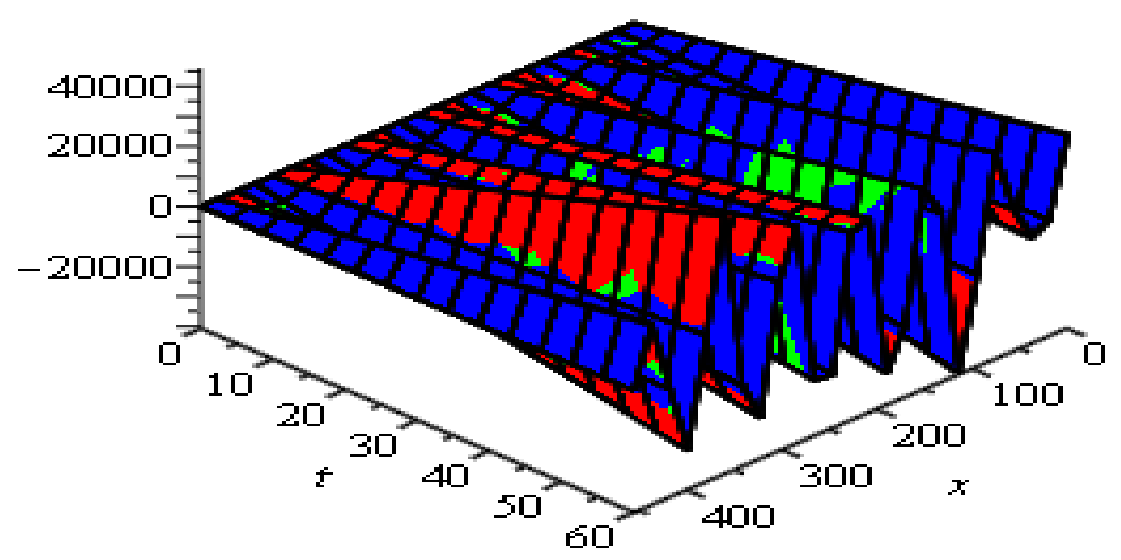

figlne 8: variation of velocity

$\mathrm{u}_{2}(x, y, f)$ wifh fine horizonfal direction $-x$ and time $\mathrm{t}$

for different values of $f$ 
Figure $7 \& 8$ shows the flow velocity of the first layer $u_{1}(x, y, t)$ and $u_{2}(x, y, t)$ in the horizontal $x$-direction and at different times $t$. The flow is irregular and random at different points in time and space. The figures described an overturned (that is inverted) character. The nature of the overturning regions is strongly dependent on the strength of stratification which caused vertically layered flow structures in stratified turbulent fluid thereby producing local shear instabilities due to sharp vertical gradients and subsequently overturning.

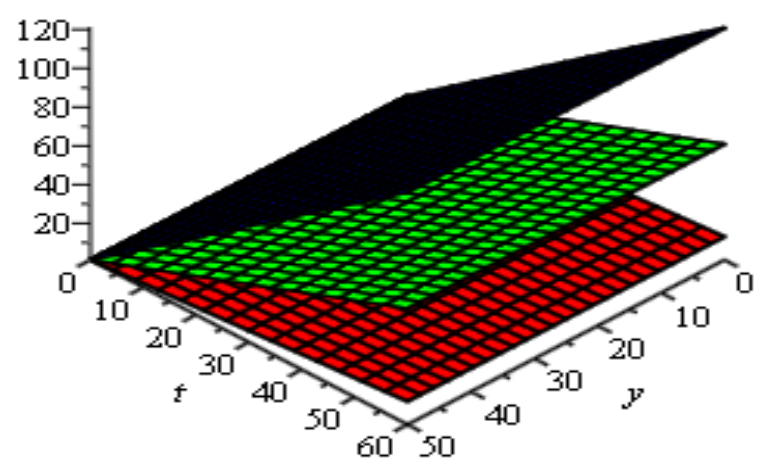

figure 9: variation of velocity $u_{1}(x, y, t)$ with the horizontal direction $-y$ and time $\mathrm{t}$ for different values of $f$

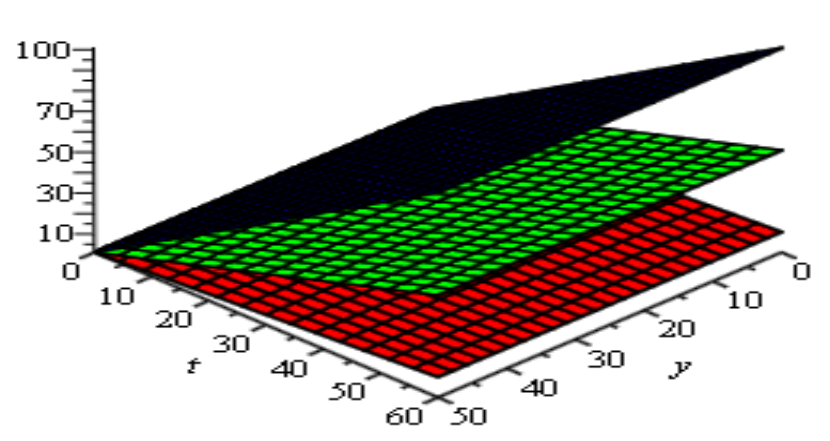

figure 10: variation of velocity $u_{2}(x, y, f)$ with the horizontal direction $-y$ and time $\mathrm{t}$ for different values of $f$

Figure 9 and 10 illustrates the flow velocity $u_{1}(x, y, t)$ and $u_{2}(x, y, t)$ in the stratified fluid in $y$-direction at different times. Different colour indicates differents value of $f$ showing a plane with deformable boundary within them. 

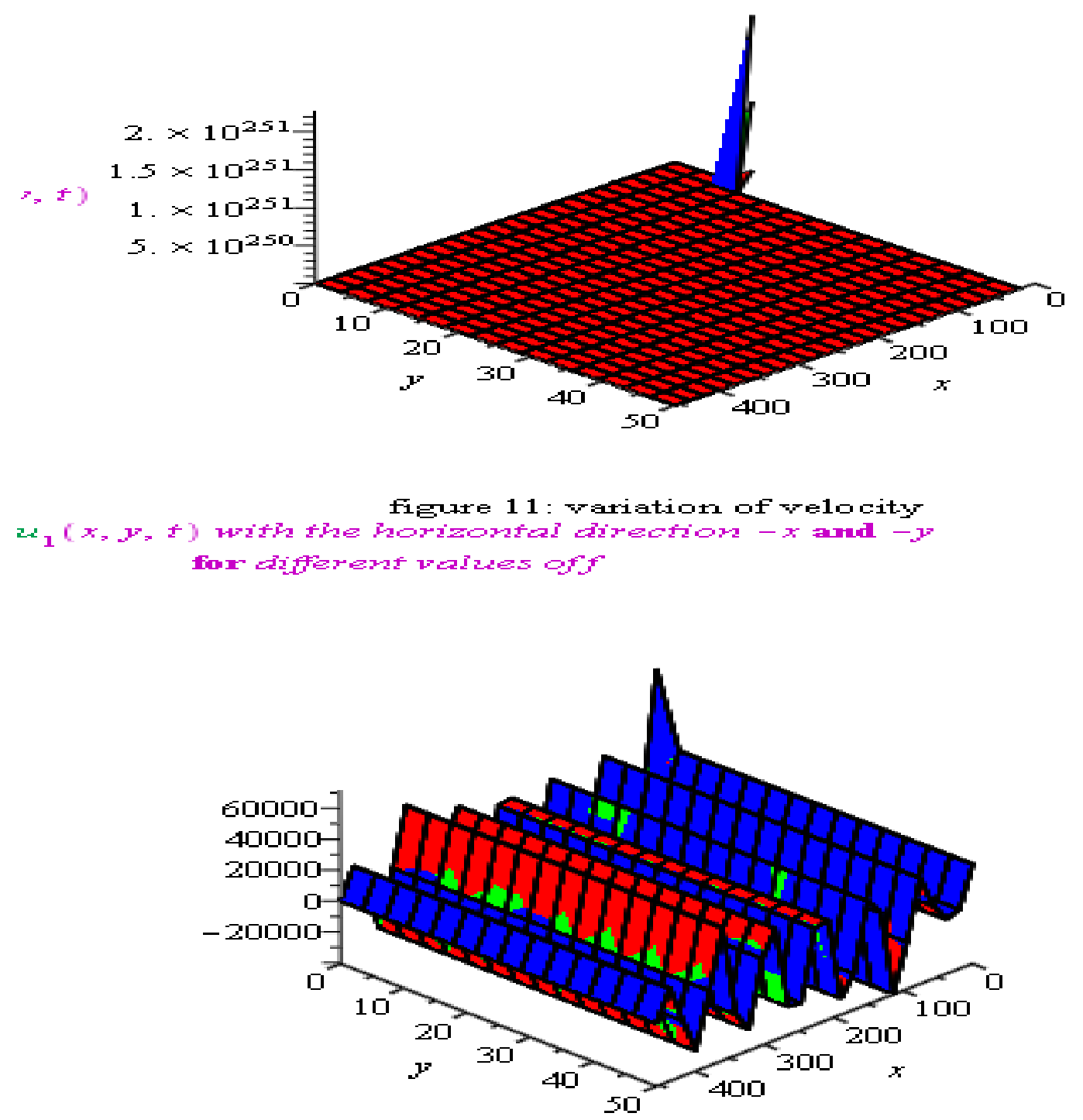

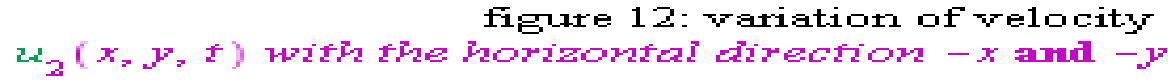
for duterent paldes off
}

Figure 11 shows the flow velocity $u_{1}(x, y, t)$ in the horizontal $x-y$ directions of the stratified flow. The surface of the flow thus formed is called a free surface because the flow boundary is freely deformable in contrast to the solid boundaries. The effect of the bottom topography causes the water to shoot up above the normal water surface. Figure 12 shows the flow velocity $u_{2}(x, y, t)$ in the second layer of the stratified fluid where the fluid density is higher than in the first layer. The nature of overturning regions is shown to be strongly dependent on the strength of stratification and the bottom topography. 


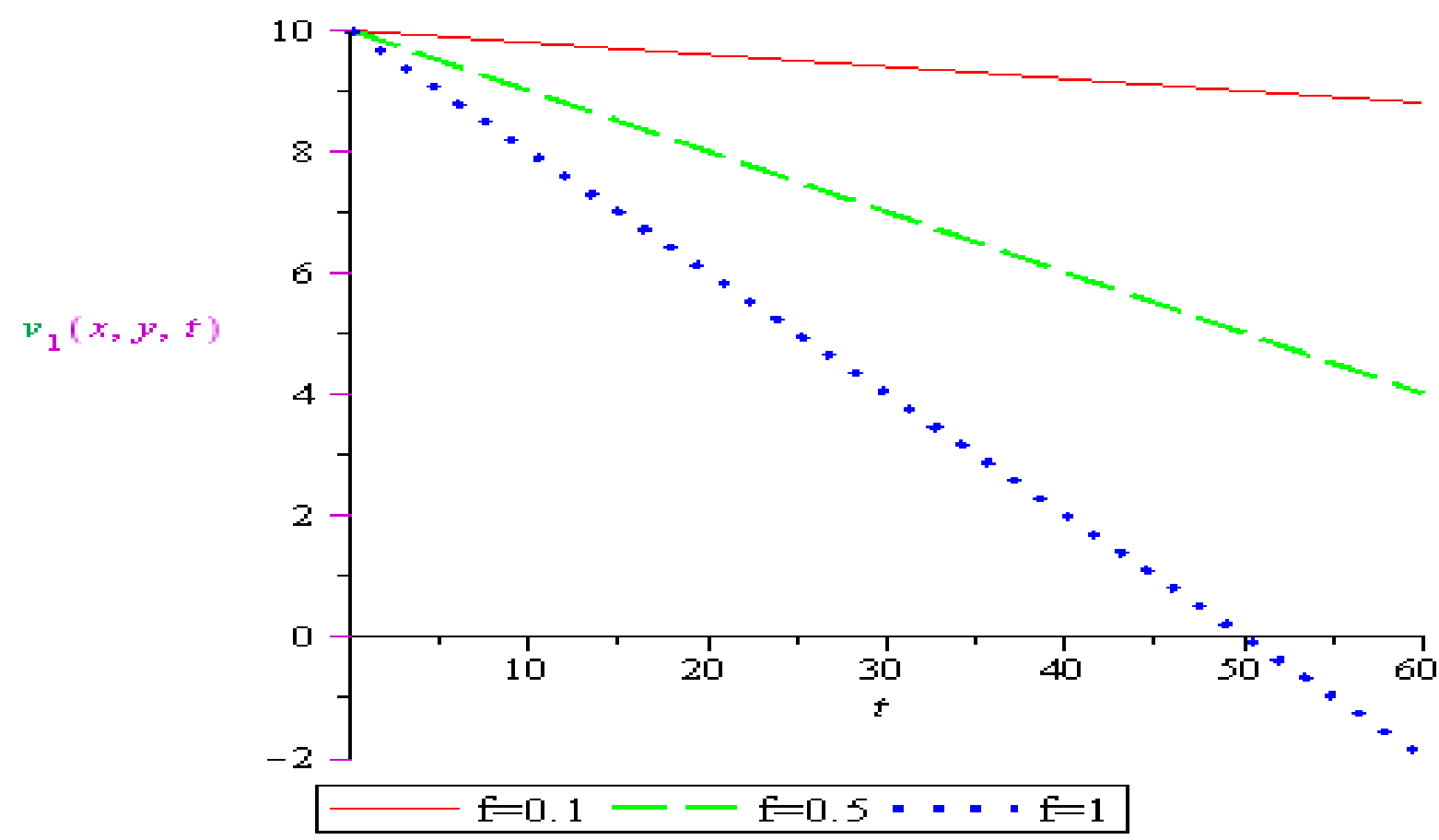

figure 13 variation of $v e 10$ city $v_{1}(x, y, t)$ with distatuce $y$, with different values of $f$.

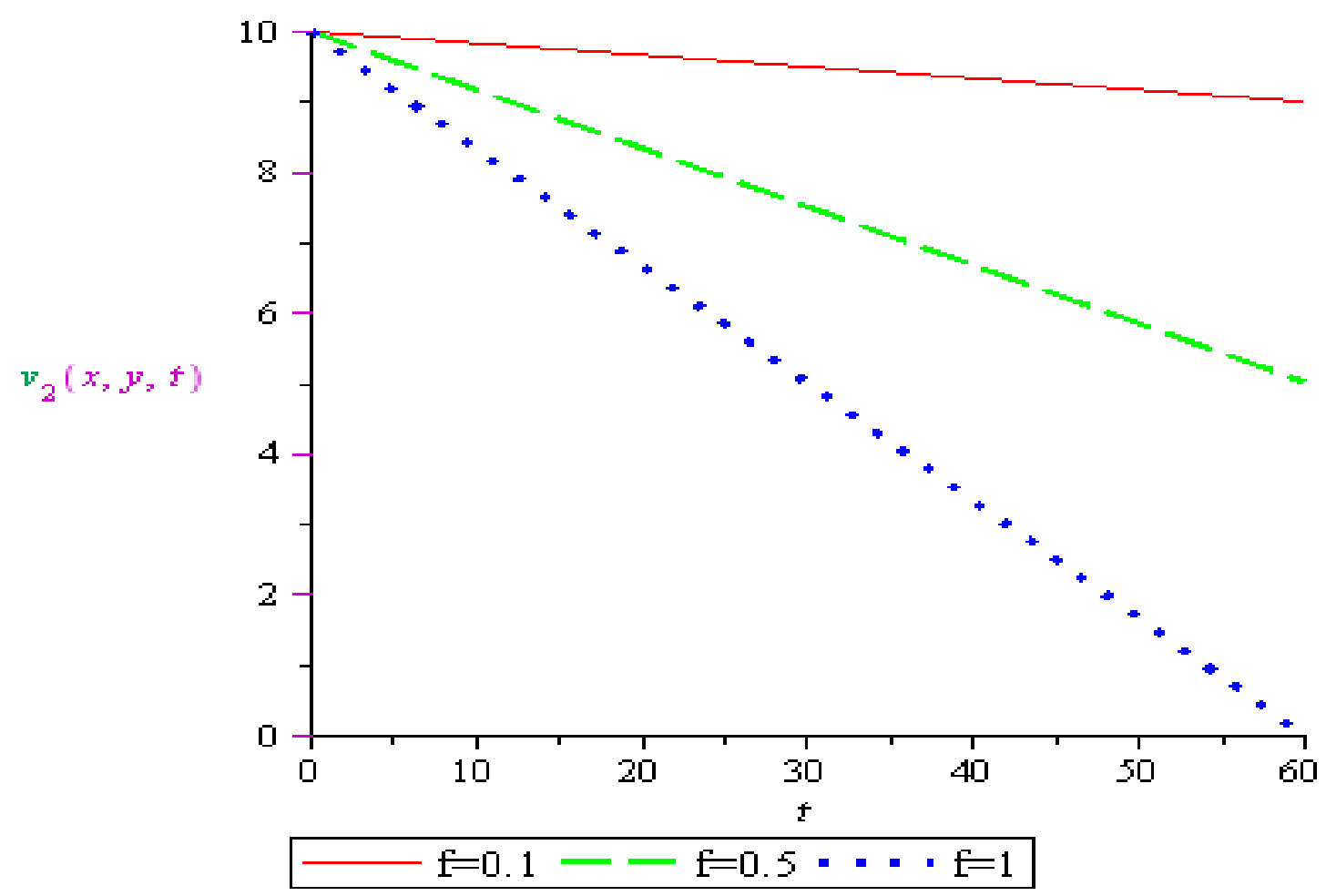

figure 14 variation of velocity $v_{2}(x, y, t)$ with distance $y$, with different. values of $f$.

Figure $13 \& 14$ shows retardation of the flow rate of the velocity with time at different values of $f$. For $f=0.1$ we have a slowly varying retardation as time increases. At $f=0.5$ the retardation is faster but slower than that of $f=1$ as time increases. This shows that when the force due to rotation is high the rate of flow velocity $v_{1}(x, y, t)$ and $v_{2}(x, y, t)$ on the surface in the horizontal $y$-direction will be very fast and may reduce to zero. 


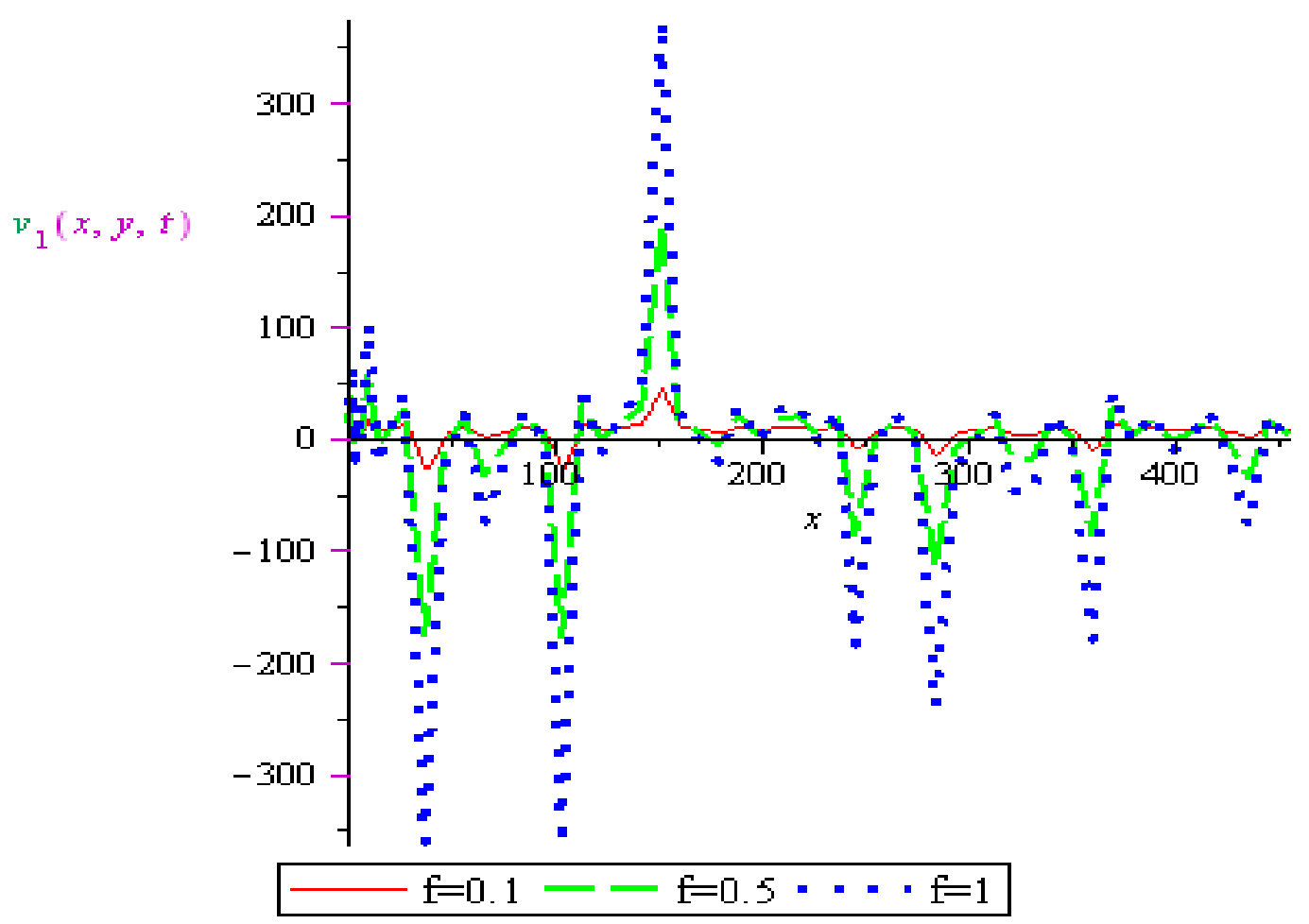

figure 15 variation of velocity $v_{1}(x, y, t)$ with distance $x$, with different. values of $f$.

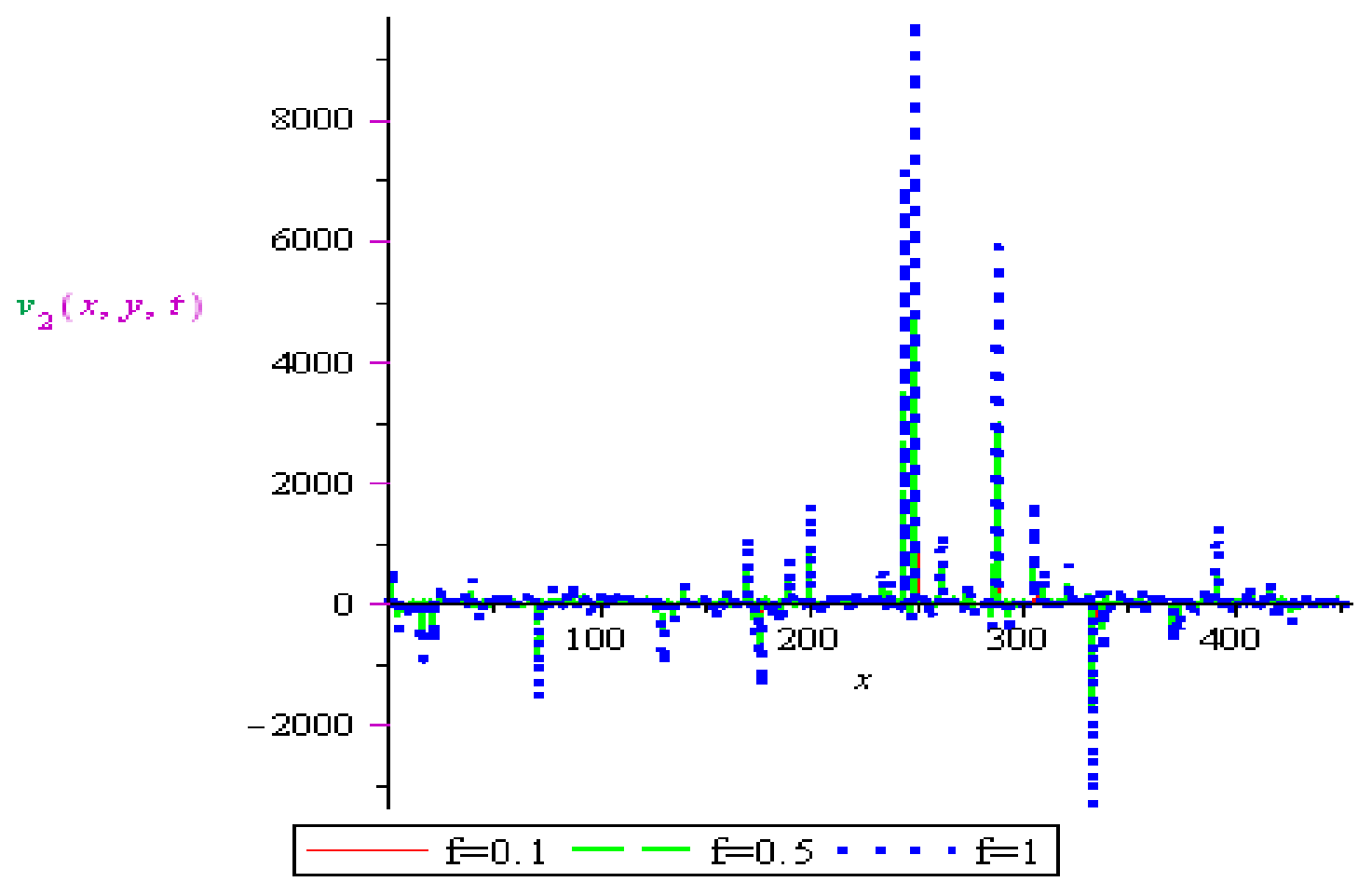

figure 16 variation of velocity $v_{2}(x, y, t)$ with distance $x$, with different values of $\mathrm{f}$.

Figure $15 \& 16$ shows the variation of flow velocity $v_{1}(x, y, t)$ and $v_{2}(x, y, t)$ in the horizontal $x$-direction with different values of $f$.The flow in the second layer which is closer to the bottom topopgraphy have more obstructions that causes loss in momentum than 
in the first layer. The obstructed waves inturn become barriers to other incoming waves which then causes more congestion in the second layer than in the first layer thereby decreasing the rate of flow velocity across the water bed and thus resulting in the disorderliness and turbulence in flow as shown.

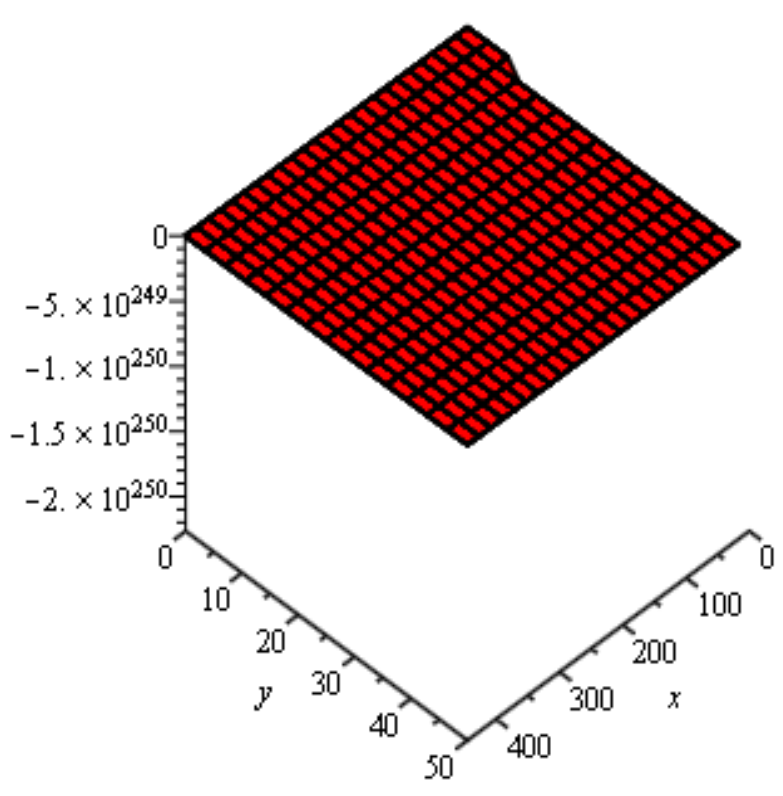

figure 17: variation of velocity $v_{1}(x, y, t)$ with the horizontal direction $-\mathrm{y}$ and $-\mathrm{x}$ for different values of $f$

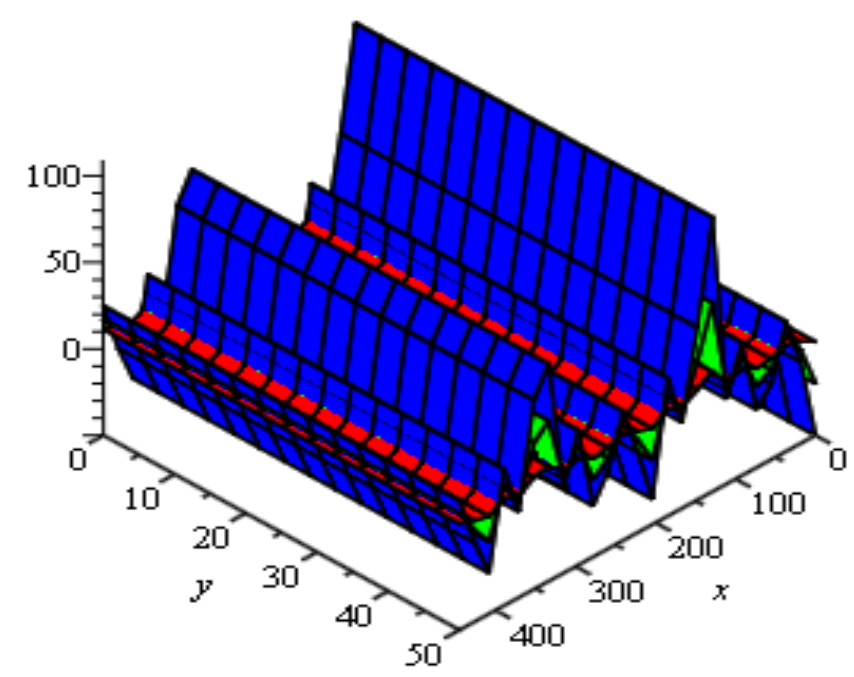

figure 18: variation of velocity $v_{2}(x, y, f)$ with the horizontal direction $-y$ and $-x$

for different values of $f$ 
Figure 17 shows the variation of flow velocity $v_{1}(x, y, t)$ of the first layer of fluid in the $x-y$ directions and at different values of $f$ and this shows a plane surface flow in 2-D directions. The negative velocity values indicates that the flow is imaginary. Figure 18 shows the variation of the flow velocity $v_{2}(x, y, t)$ of the second layer of fluid in the $x-y$ directions and at different values of $f$ which demonstrates that due to the obstruction of waves both on the bottom and the side boundaries respectively are barriers to other incoming waves and thus causes an interwoven of the waves and overturn in the flow.

\section{Conclusion}

We were able to derive the mathematical models for the stratified geophysical fluid flow over variable bottom topography. We obtained a system of six nonlinear partial differential equations in twodimensions with Coriolis force and bottom topography included and these were solved using perturbation method. The presence of the Coriolis force in the shallow water equations were shown to cause the deflection of fluid parcels in the direction of wave motion and causes gravity waves to disperse. As water depth decreases due to bottom topography, the wave amplitude increases, the wavelength and wave speed decreases resulting in overturning of the wave. The effect of the Coriolis force is seen in the oscillatory motion in the direction of wave motion which causes gravity waves to disperse.

Fluids in the geophysical flow are generally in motion. The surface resistance in the planetary boundary layer or the wind driven force of the rivers and oceanic surface layer means that the motion varies with amplitude. Despite the stable density stratification, the velocity varies adequately fast with height, destabilizing the flow and resulting in violent overturning of the density surfaces. Geophysical fluid flow with varied bottom topography strongly influences stratified flows because fluid parcels are typically deflected in both the horizontal and vertical direction by the obstacle. This behavior is parameterized by the Froude number. The Froude number is a measure of the likelihood of the flow to be deflected vertically against the density stratification. However, the response of a stratified flow to topography is also dependent upon the shape of the obstacle, but for simple two dimensional obstacles, our study has found regimes in which violent overturning of density surfaces can occur.

In addition, the vertical deflections of fluid parcels generate internal waves which can propagate vertically in the stratified flow. However, owing to the variation of velocity and stratification with height in the shallow water, it is likely that these waves will break and form regions of turbulent overturning at some level.

\section{References}

Abd-el-Malek M. B., Badran N. A. and Hassan H. S.(2007). "Lie-Group Method for Predicting Water Content for Immiscible Flow of Two Fluids in a Porous Medium," Applied Mathematical Sciences, Vol. 1, No. 24, pp. 1169-1180.

Blumberg, A.F., Herring, H.J. (1987). Circulation modelling using orthogonal curvilinear coordinates. In: Nihoul, J.C.J., Jamart, B.M. (eds) Threedimensional Models of Marine and Estuarine Dynamics. Elsevier Oceanography Series, Amsterdam

Boyer, D.L., Davies, P.A. (2000). Laboratory studies of orographic effects in rotating and stratified flows. Annu. Rev. Fluid Mech., 32, 165-202.

Dellar P. J. and Salmon R. (2005). "Shallow Water Equations with a Complete Coriolis Force and Topography," Physics of Fluids, Vol. 17, No. 10, , pp. 106601-106619.

Gill, A.E.: Atmosphere-Ocean Dynamics. Academic Press, New York London Sydney Tokyo Toronto (1982).

Iornumbe S.I., Mbah G.C.E., and Chia R.A. (2020). Mathematical Model of Geophysical Fluid Flow over Variable Bottom Topography, Nigerian Annals of pure and applied Sciences, Volume 3(2). 163 
Karelsky K. V., Papkov V. V., Petrosyan A. S. and Tsygankov D. V. (2000). "Particular Solution of the ShallowWater Equations over a Non-Flat Surface," Physics Letters A, Vol. 271, No. 5-6, pp. 341-348.

Lynch, D.R., Werner, F.E. (1991).Threedimensional hydrodynamics on finite elements. Part II: Non-linear timestepping model. Int. J. Numer. Methods Fluids, 12,

507-533.

Mellor, G.L. (1996). Introduction to Physical Oceanography. Springer, New York Berlin Heidelberg

Mellor, G.L., Yamada, T. (1982).Development of a turbulence closure model for geophysical fluid problems. Pev. Geophys. Space Phys., 20, 851-875.

Moe, H., Ommundsen, A., Gjevik, B. (2002).A high resolution tidal model for the area around The Lofoten Islands, northern Norway. Continental Shelf Res. 22, 485-504.
Pedlosky, J. (1987). Geophysical Fluid Dynamics. Springer, New York Heidelberg Berlin London Paris Tokyo Perks, C., "Dealing with stratification within a water supply reservoir", In 69th Annual Water Industry Engineers and Operators' Conference (pp. 3036).2006.

Riley, J.J., Lelong, M.P. (2000). Fluid motions in the presence of strong stable stratification. Annu. Rev. Fluid Mech., 32, 613-657.

Stull, R.B. (1988). An Introduction to Boundary Layer Meteorology. Kluwer Academic Publishers, Dordrecht Boston London

Utnes, T., Brors, B. (1993).Numerical modelling of 3-D circulation in restricted waters. Applied Math. Modelling, 17, 1-14.

Vallis, G.K. (2006). Atmospheric and oceanic fluid dynamics: fundamentals and large scale circulation. Cambridge University Press, Cambridge, 